\title{
Doping Graphene with Substitutional Mn
}

Pin-Cheng Lin, ${ }^{\dagger, \nabla}$ Renan Villarreal, ${ }^{*} \dagger, \nabla$ Simona Achilli, ${ }^{\ddagger}$ Harsh Bana, ${ }^{\dagger}$ Maya N. Nair, " Antonio Tejeda, ${ }^{\S}$ Ken Verguts,,$\perp$ Stefan De Gendt,,$\perp$ Manuel Auge,\# Hans Hofsäss, $\#$ Steven De Feyter, ${ }^{@}$ Giovanni Di Santo, ${ }^{\triangle}$ Luca Petaccia, ${ }^{\triangle}$ Steven Brems, $\|$ Guido Fratesi, ${ }^{\ddagger}$ and Lino M. C. Pereira*, ${ }^{*}$

$\dagger$ †uantum Solid State Physics, KU Leuven, 3001 Leuven, Belgium

$\ddagger$ ETSF and Dipartimento di Fisica "Aldo Pontremoli", Università degli Studi di Milano, Via Celoria, 16, I-20133 Milano, Italy

ฯCUNY Advanced Science Research Center, 85 St. Nicholas Terrace, New York, New York 10031, USA

$\S$ Laboratoire de Physique des Solides, CNRS, Univ. Paris-Sud, 91405 Orsay, France

\|imec vzw (Interuniversitair Micro-Electronica Centrum), 3001 Leuven, Belgium $\perp$ Department of Chemistry, Division of Molecular Design and Synthesis, KU Leuven, 3001 Leuven, Belgium

\#II.Institute of Physics, University of Göttingen, 37077 Göttingen, Germany @ Department of Chemistry, Division of Molecular Imaging and Photonics, KU Leuven, 3001 Leuven, Belgium

$\triangle$ Elettra Sincrotrone Trieste, Strada Statale 14 km 163.5, 34149 Trieste, Italy $\nabla$ P.-C.L. and R.V. contributed equally to this work

E-mail: renan.villarreal@kuleuven.be; lino.pereira@kuleuven.be

\begin{abstract}
We report the incorporation of substitutional $\mathrm{Mn}$ atoms in high-quality, epitaxial
\end{abstract}


graphene on $\mathrm{Cu}(111)$, using ultra-low energy ion implantation. We characterize in detail the atomic structure of substitutional $\mathrm{Mn}$ in a single carbon vacancy and quantify its concentration. In particular, we are able to determine the position of substitutional Mn atoms with respect to the Moiré superstructure (i.e. local graphene-Cu stacking symmetry) and to the carbon sublattice; in the out-of-plane direction, substitutional Mn atoms are found to be slightly displaced towards the $\mathrm{Cu}$ surface, i.e. effectively underneath the graphene layer. Regarding electronic properties, we show that graphene doped with substitutional Mn to a concentration of the order of $0.04 \%$, with negligible structural disorder (other than the Mn substitution), retains the Dirac-like band structure of pristine graphene on $\mathrm{Cu}(111)$, making it an ideal system in which to study the interplay between local magnetic moments and Dirac electrons. Our work also establishes that ultra-low energy ion implantation is suited for substitutional magnetic doping of graphene; given the flexibility, reproducibility and scalability inherent to ion implantation, our work creates numerous opportunities for research on magnetic functionalization of graphene and other 2D materials.

\section{Keywords}

graphene, doping, manganese, electronic structure, magnetism, ion implantation

Magnetic functionalization of graphene is being intensively investigated for its fundamental appeal as well as for applications in next-generation electronics. ${ }^{1-8}$ The formation of local magnetic moments and their interactions in graphene, which is intrinsically diamagnetic and exhibits electronic behavior beyond that of conventional metals and semiconductors, remains however poorly understood. Harnessing such magnetic properties would enable various applications in spintronics and related technologies.

So far, magnetic functionalization of 2D materials has been explored by introducing magnetic moments in different forms, including intrinsic defects such as $\mathrm{C}$ vacancies, ${ }^{1}$ hydrogenation, ${ }^{2}$ adatoms (e.g. transition metals ${ }^{3,9,10}$ and rare earths ${ }^{4}$ ), proximity to full magnetic 
layers ${ }^{5}$ and magnetic molecules. ${ }^{6}$ However, these approaches present various limitations in terms of stability against ambient temperature and atmosphere (e.g. segregation and oxidation) and of scalable device fabrication, which constitute major difficulties not only in an application scenario but also for basic research. Substitutional doping, ${ }^{11,12}$ on the other hand, offers high stability thanks to the covalent bonding between the dopant atom and the surrounding carbon atoms in graphene.

Substitutional doping with non-magnetic elements such as N and B can be achieved with relatively simple processes, even during growth. ${ }^{13,14}$ Substitutional doping with typical impurities such as $\mathrm{Si}$ (present during growth or processing) has also been observed. ${ }^{15-17}$ However, substitutional incorporation of magnetic elements such as transition metals is generally not thermodynamically favored due to their different chemical nature compared to C. Substitutional doping with transition elements ${ }^{11,12,18}$ has been achieved using non-equilibrium conditions: first, vacancies are formed by bombardment with electrons or ions, and subsequently the magnetic dopant atoms are deposited, filling the vacancies that have not dynamically annealed between the two steps. Such dual-step processes are difficult to control, especially given the complex dynamics of vacancies in graphene, thus strongly limiting their reliability, reproducibility and scalability. Ultra-low energy (ULE) ion implantation has the potential to overcome these limitations and has been successfully applied to the substitutional doping of graphene with $\mathrm{N}$ and $\mathrm{B},{ }^{19-23} \mathrm{P}^{24}$ and Ge. ${ }^{25}$

Here, we report the incorporation of substitutional Mn atoms in graphene using ULE ion implantation. We selected $\mathrm{Mn}$ as a model case of magnetic doping, since it is expected to exhibit the largest magnetic moment among $3 d$ transition metals in a single $\mathrm{C}$ vacancy, ${ }^{26}$ which is typically advantageous for magnetic functionality. We provide a detailed characterization of the atomic structure of substitutional Mn in graphene, based on scanning tunneling microscopy (STM) and density functional theory (DFT) calculations. Furthermore, using angle-resolved photoemission spectroscopy (ARPES), we show that graphene doped with substitutional Mn retains the Dirac-like behavior of pristine graphene. These findings mo- 
tivate further research on magnetic functionalization of 2D materials, strongly benefiting from the high stability of substitutional doping, together with the high degree of control, flexibility and scalability of ultra-low energy ion implantation.

\section{Results and Discussion}
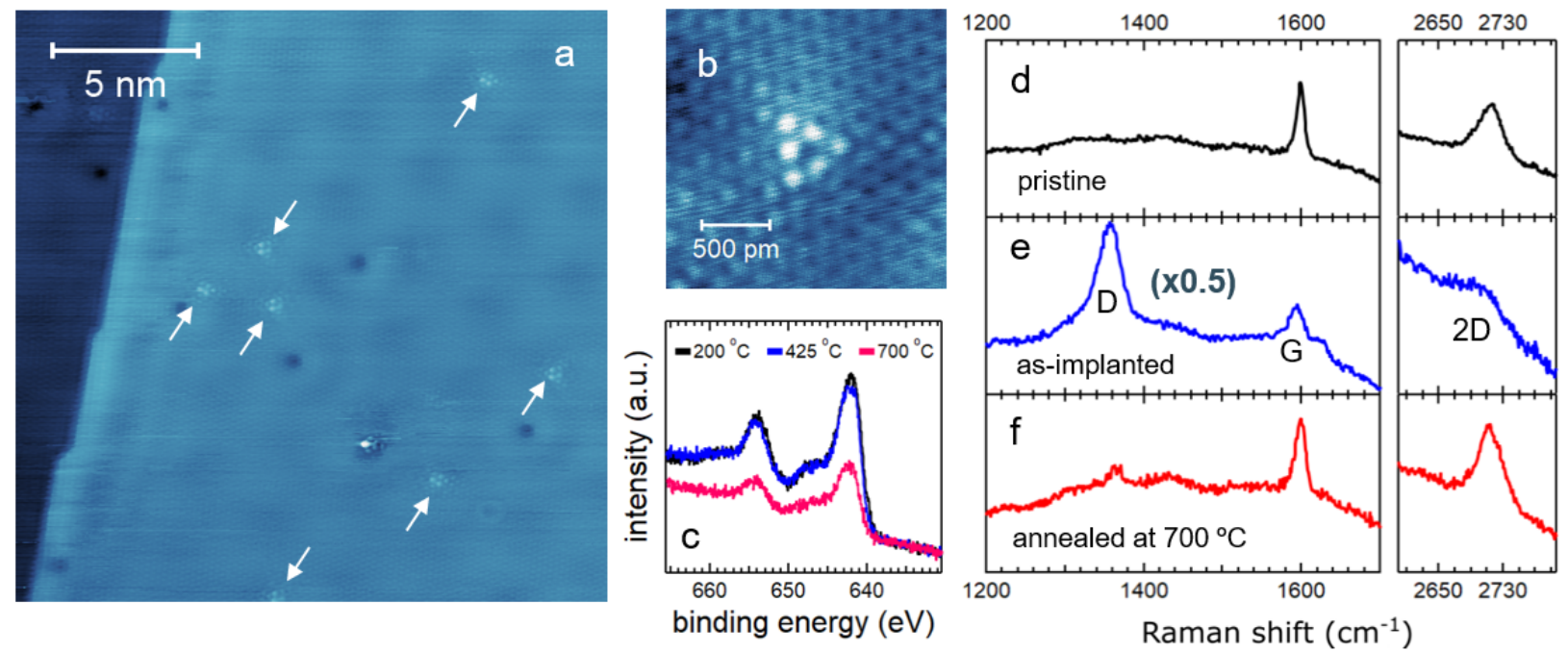

Figure 1: STM micrographs of Mn implanted graphene on $\mathrm{Cu}(111)$ after $700{ }^{\circ} \mathrm{C}$ annealing: (a) large-scale micrograph showing substitutional Mn defects pointed by white arrows $\left(V_{\text {sample }}=200 \mathrm{mV}, I_{\text {tun. }}=300 \mathrm{pA}\right)$; (b) small-scale micrograph showing one substitutional Mn defect $\left(V_{\text {sample }}=-100 \mathrm{mV}, I_{\text {tun }}=5 \mathrm{nA}\right.$ ). (c) Mn 2p core-level spectrum of Mn implanted graphene $/ \mathrm{Cu}(111)$, annealed at the following temperatures after implantation: 200 ${ }^{\circ} \mathrm{C}$ (black), $425{ }^{\circ} \mathrm{C}$ (blue) and $700{ }^{\circ} \mathrm{C}$ (pink). (d-f) Raman spectra (laser wavelength $=532$ $\mathrm{nm}$ ) on graphene/Cu(111): (d) pristine sample annealed at $700{ }^{\circ} \mathrm{C}$; (e) Mn implanted, in the as-implanted state; (f) $\mathrm{Mn}$ implanted and annealed at $700{ }^{\circ} \mathrm{C}$ after implantation. The positions of the D, G and 2D bands of graphene are indicated in panel (e).

Sample preparation and basic characterization. The samples consist of epitaxial graphene grown by chemical vapor deposition (CVD) on epitaxial $\mathrm{Cu}(111)$ thin films grown on sapphire(0001) substrates as described in Ref. 27. ULE ion implantation was performed by electrostatic deceleration of a ${ }^{55} \mathrm{Mn}^{+}$ion beam from $30 \mathrm{keV}$ to $40 \mathrm{eV}$, with perpendicular incidence with respect to the sample surface, to a fluence of $1.5 \times 10^{14}$ ions per $\mathrm{cm}^{2}$ (fluence rate of $\left.1.7 \times 10^{11} \mathrm{~cm}^{-2} \cdot \mathrm{s}^{-1}\right)$ measured by integration of the electric current on sample 
during implantation. ${ }^{19,21,22}$ The samples were subsequently studied in the as-implanted state as well as after thermal annealing under ultra-high vacuum UHV (below $10^{-9}$ mbar) up to $700{ }^{\circ} \mathrm{C}(20 \mathrm{~min})$. This article focuses on the $700{ }^{\circ} \mathrm{C}$ annealed state, where STM measurements provide the clearest identification (compared to the other annealing stages) of $\mathrm{Mn}$ defects that, according to the analysis described in the following paragraph, we identify as substitutional Mn atoms in graphene. Moreover, this STM-based identification allows us to quantify its concentration as $0.04 \pm 0.02 \%$ with respect to $\mathrm{C}$ atoms in graphene (Figure 1 (a, b) and Table S1), which corresponds to approximately $1 \%$ of the implanted ions (i.e. $1 \%$ of the implanted $1.5 \times 10^{14}$ ions per $\mathrm{cm}^{2}$ ) being substitutionally incorporated. The remainder corresponds to Mn ions in other configurations: backscattered during implantation (i.e. back into the vacuum upon collision with $\mathrm{C}$ and $\mathrm{Cu}$ atoms), adsorbed onto graphene, intercalated between graphene and the $\mathrm{Cu}$ surface, or diffused into $\mathrm{Cu}$ upon annealing. Nonsubstitutional configurations are also observed in the STM data, although not as clearly identified (Figure S3). Synchrotron X-ray photoelectron spectroscopy (XPS) measurements reveal a decrease in $\mathrm{Mn}$ concentration (with respect to $\mathrm{C}$ in the graphene layer) upon annealing: from $2.3 \%$ and $2.2 \%$ after thermal annealing at $200{ }^{\circ} \mathrm{C}$ and $425^{\circ} \mathrm{C}$, respectively, to $1.0 \%$ after annealing at $700{ }^{\circ} \mathrm{C}$ (Figure $1(\mathrm{c})$, additional details are given in supplementary information). We did not determine the Mn concentration in the as-implanted state, i.e. asmounted in the UHV chamber after exposure to ambient conditions, since in that state the surface is inherently highly contaminated, hindering quantification and possibly modifying the sample surface upon photon irradiation (more details in supplementary information). The Mn concentration at low annealing temperature $\left(2.3 \%\right.$ for at $\left.200{ }^{\circ} \mathrm{C}\right)$ corresponds to about half of the Mn fluence $\left(1.5 \times 10^{14} \mathrm{~cm}^{-2}\right)$, indicating that approximately half of the implanted ions are in fact backscattered (lost to the vacuum) already during the implantation process (but still deposit their charge, thus being accounted in the fluence value). The further decrease upon annealing (down to $1 \%$ at $700{ }^{\circ} \mathrm{C}$ ) can be understood as due to desorption of Mn atoms (i.e. loss of Mn to the vacuum) which had initially adsorbed onto 
graphene during implantation, and diffusion of intercalated Mn deeper into the Cu layer (i.e. the Mn atoms remain in the sample but their apparent intensity decreases due to inelastic scattering of the photoelectrons). Thermal annealing also removes adsorbed contaminants resulting from exposure to air between the multiple synthesis and characterization steps (cf. Table S3 and Table S4). Raman spectroscopy (Figure 1(d-f)) shows onset of significant disorder upon implantation (e), with the emergence of a D peak with a peak-height ratio with respect to the $\mathrm{G}$ peak $\left(I_{\mathrm{D}} / I_{\mathrm{G}}\right)$ of 2.14 , whereas in the pristine sample $(\mathrm{d})$ no $\mathrm{D}$ peak is observed. A low-disorder state is recovered upon annealing at $700{ }^{\circ} \mathrm{C}\left(I_{\mathrm{D}} / I_{\mathrm{G}}=0.19\right)$. The residual D peak intensity is likely related to the substitutional Mn defects, although other defects (formed upon implantation or annealing) cannot be excluded. In short, the (partial) removal of non-substitutional Mn components, combined with a cleaner graphene surface and low disorder, make the $700{ }^{\circ} \mathrm{C}$ annealed state particularly suitable for a more detailed study of substitutionally doped graphene, which constitutes the remainder of this article.

Atomic-scale structure of substitutional Mn. By combining STM measurements and DFT simulations, we are able to analyse the atomic-scale structure of substitutional Mn in extreme detail. In the following, we present the main findings regarding the identification and structure of the defects. Further details are given in supplementary information. We must first consider the stacking between the graphene lattice and the $\mathrm{Cu}(111)$ surface. As a result of the different lattice parameter $(2.46 \AA$ for graphene and $2.56 \AA$ for the $\mathrm{Cu}$ surface $)$ and of the angular orientation of graphene with respect to the $\mathrm{Cu}$ surface $\left( \pm 3.57^{\circ}\right.$, determined using low-energy electron diffraction, $c f$. Figure S6(b)), the graphene-Cu stacking varies continuously across the surface (Figure 2(a)) leading to a Moiré superstructure (e). Three points with high-symmetry stacking can be defined indicating the position of the two C atoms in the graphene unit cell with respect to the underlying substrate: TOP-FCC (Figure 2(b)) TOP-HCP (c), HCP-FCC (d). Between these points the stacking has lower symmetry. These high-symmetry points define a periodic 2-dimensional lattice, which is observed in the STM 
topographies as a Moiré superstructure (Figure 2(e)). The identification of the three highsymmetry stacking regions in the STM topographies (Figure 2(e)) is based on the simulated Cu-graphene distances $(2.60 \AA$ for TOP-FCC, $2.70 \AA$ for TOP-HCP, and $2.87 \AA$ for HCPFCC), which is reflected in the STM data as height differences, i.e. lowest for TOP-FCC and highest for HCP-FCC (Figure S7). For a given high-symmetry stacking, and assuming that only one $\mathrm{C}$ vacancy is available, $\mathrm{Mn}$ can substitute for one of two non-equivalent $\mathrm{C}$ atoms. For example, for TOP-FCC stacking, Mn can substitute for the $\mathrm{C}$ atom that is vertically aligned either with the TOP site or the FCC site of the Cu surface (Figure 3). We performed DFT calculations for all six combinations of three stackings and two sites.

Furthermore, by comparing DFT-simulated and experimental STM topographies, we identify the surface structure of $\mathrm{Mn}$ substituting for a single $\mathrm{C}$ atom, i.e. located at a single $\mathrm{C}$ vacancy (Figure 3). This unambiguous identification is based not only on the comparison to simulations of substitutional $\mathrm{Mn}$ in a single vacancy, but also to simulations of a wide range of other defects, intrinsic or Mn-related: free single vacancies, free double vacancies, Mn in double vacancies, intercalated Mn (without vacancy), all of which can be distinguished from substitutional Mn in a single vacancy (Figure S1). Some of these other defects are indeed observed in our samples (Figure S3), although their identification is more ambiguous than that of substitutional Mn, which is the focus of the present article. We also note that we observe substitutional Mn defects already in the as-implanted state; however, as mentioned above, given the cleaner surface and lower degree of disorder, here we focus on the $700{ }^{\circ} \mathrm{C}$ annealed state. For all the six analyzed substitutional configurations, we find that the STM simulations reproduce the shape and contrast observed in the STM measurements. The TOP-FCC case is illustrated in Figure 3; additional simulations are shown in Figure S2. By referring to our structural model (Figure 3(d-g)) we are able to identify the six protrusions producing the characteristic triangular symmetry as the carbon atoms from the sublattice opposite to the vacancy sublattice (corresponding to the substitutional Mn site), with the three nearest neighbours to the substitutional Mn exhibiting the highest intensity. 
In contrast, the Mn site appears as a dark region, consistent with the lower position of the Mn atom with respect to the surrounding $\mathrm{C}$ atoms. In Figure 3(a), we exhibit in particular two substitutional Mn defects in opposite graphene sublattices, showing triangular features which are mirror reflected with respect to each other, again in agreement with the symmetry relation obtained in the DFT simulations (Figure $3(\mathrm{~b}, \mathrm{c})$ ). The site identification of the Mn defects in Figure 2(e) is based on this relation between stacking region, Mn site (i.e. C sublattice), and orientation of the triangular-shaped objects in the STM data; no other differences were observed in the topographic signatures for substitutional $\mathrm{Mn}$ in different stacking regions.
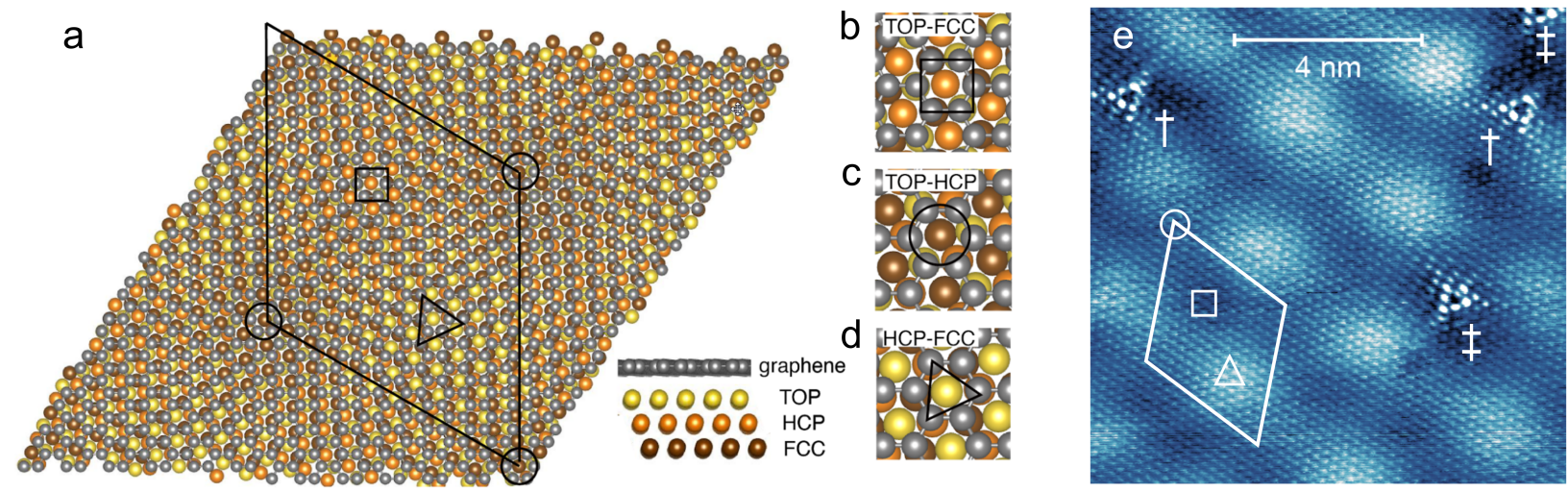

Figure 2: (a) Moiré superstructure of graphene/Cu(111), resulting from the different graphene-Cu stacking regions: (b) TOP-FCC, (c) TOP-HCP, (d) HCP-FCC. (e) STM micrograph $\left(V_{\text {sample }}=100 \mathrm{mV}, I_{\text {tun }}=100 \mathrm{pA}\right)$ showing the Moiré superstructure and substitutional Mn defects within them: TOP-FCC stacking with Mn on the fcc site (marked with $\dagger$ ); TOP-HCP stacking with Mn on the HCP site (marked with $\ddagger$ ). The identification of the stacking region and of $\mathrm{Mn}$ site is described in the main text. Symbols $(\bigcirc, \square, \triangle)$ are overlaid to link the identification of the three stacking regions in the various panels.

A particularly important aspect of the atomic structure of substitutional $\mathrm{Mn}$ is the vertical displacement with respect to the graphene layer (Figure 3(e, g)). Due to the short bond-lengths that characterize graphene, and to the large effective radius of Mn compared to the free space left by the vacancy, substitutional Mn atoms relax away from the graphene plane (Figure 3(e, g)). This out-of-plane displacement has been proposed to occur for various substitutional dopants in graphene $\left(\right.$ e.g. $\left.\mathrm{P}^{24}\right)$. However, previous studies on such 


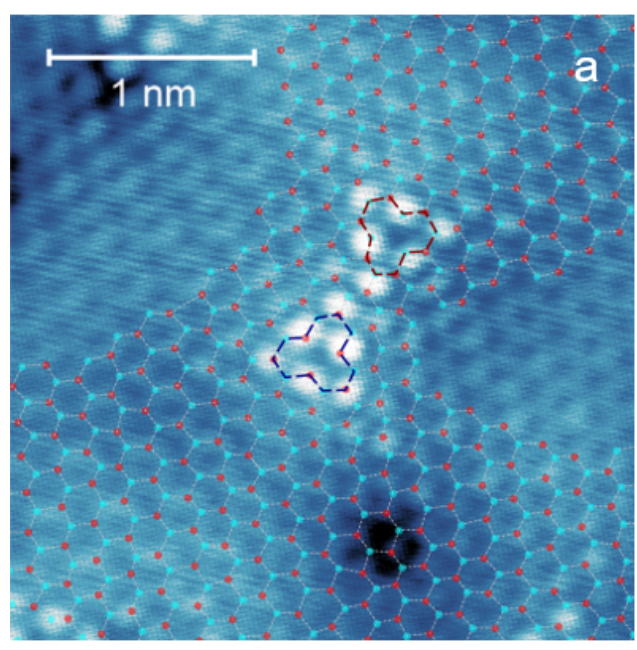

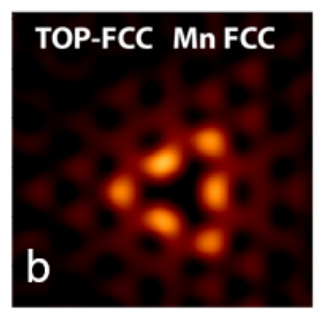

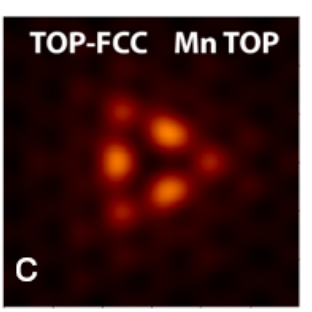

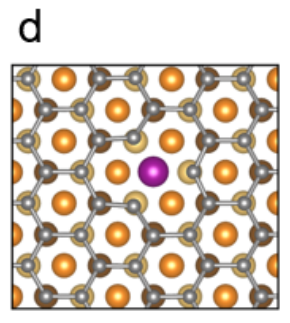
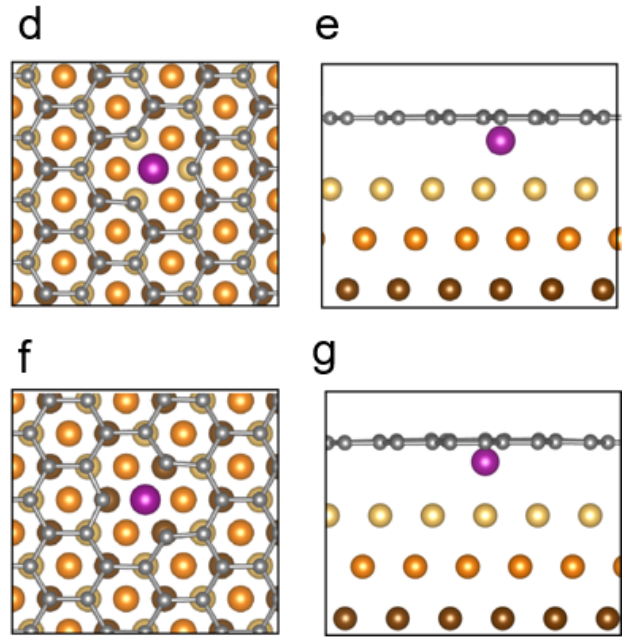

g

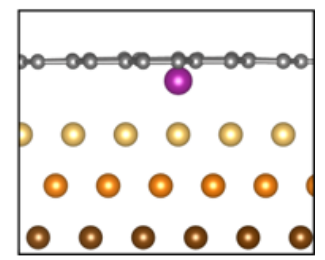

Figure 3: (a) STM micrograph $\left(V_{\text {sample }}=-100 \mathrm{mV}, I_{\text {tun. }}=5 \mathrm{nA}\right)$ exhibiting two substitutional Mn defects in opposite graphene sublattices, showing triangular features which are mirror reflected with respect to each other. DFT simulated STM for substitutional Mn in a TOP-FCC stacking region (energy window $\left[-0.25 \mathrm{eV}, \mathrm{E}_{\mathrm{F}}\right]$ ): (b) for $\mathrm{Mn}$ in $\mathrm{FCC}$ site; (c) in TOP site. Relaxed model structures obtained from DFT: (d, e) top and side view, respectively, for $\mathrm{Mn}$ in FCC site; ( $\mathrm{f}, \mathrm{g}$ ) top and side view, respectively, for Mn in TOP site.

effects have focused on suspended graphene, which has mirror symmetry with respect to the graphene plane, before being broken by the out-of-plane displacements upon substitution. In the present work, the $\mathrm{Cu}$ substrate breaks that mirror symmetry, and we observe that $\mathrm{Mn}$ is displaced towards the $\mathrm{Cu}$ surface, while the carbon atoms surrounding the Mn atom are slightly buckled away from the $\mathrm{Cu}$ surface, with an overall corrugation not exceeding $0.1 \AA$. The $\mathrm{Mn}-\mathrm{Cu}$ distance is $\sim 2 \AA$ while the equilibrium distance between the surface and the graphene layer is $\sim 3 \AA$. Interestingly, the $\mathrm{C}$ nearest neighbours (to $\mathrm{Mn}$ ) do not retain, upon relaxation, the reconstruction that is typical of the single vacancy (taken as starting configuration); instead they symmetrically displace in plane in order to accommodate the substitutional $\mathrm{Mn}$ atom (the $\mathrm{C}-\mathrm{C}$ bond distance within the same sublattice is 2.82 $\AA$, corresponding to an increase of $11 \%$ compared to the same distance obtained far from the Mn defect). This configuration is obtained as final (relaxed state) when starting with the Mn atom just below the graphene layer. The configuration with the Mn atom above the vacancy site (protruding outwards, into the vacuum) can also be obtained as a final state 
of the DFT simulation, by placing the Mn atom exactly at the vacancy position and allowing for relaxation. For this final structural configuration (protruding outwards), the STM simulation shows a very distinct signature: bright $\mathrm{Mn}$ position versus dark surrounding $\mathrm{C}$ atoms (Figure S1(c)), i.e. the opposite of what we observe for the defects that we identify as substitutional Mn. From STM data, which is local in nature, we cannot completely exclude that the outwards-protruding configuration is also present for a minority fraction of the Mn atoms ( $c f$. one rare case in Figure S3(a) that resembles the DFT simulation in Figure S1(c)). Nevertheless, this outwards configuration is very rare, we only detected a few objects in all our STM data that may be associated with it, i.e. more than two orders of magnitude lower probability compared to the inwards configuration. This absence (or unlikely occurrence) of the outwards configuration is consistent with our DFT calculations, yielding a higher total energy (by $2.25 \mathrm{eV}$ ) compared to the inwards configuration (Figure 3(e, g)).

Table 1: Total energy difference $\Delta E_{\text {tot }}$ for substitutional Mn in different stacking-site configurations, with respect to TOP-FCC stacking with Mn in FCC site. For each configuration, the magnetic moment of $\mathrm{Mn}\left(\mu_{\mathrm{Mn}}\right)$ and of the $\mathrm{C}$ atoms $\left(\mu_{\mathrm{C}}\right)$ surrounding the vacancy (average) is also given.

\begin{tabular}{ccccc}
\hline Stacking & Site & $\Delta E_{\text {tot }}(\mathrm{eV})$ & $\mu_{\mathrm{Mn}}\left(\mu_{\mathrm{B}}\right)$ & $\mu_{\mathrm{C}}\left(\mu_{\mathrm{B}}\right)$ \\
\hline TOP-FCC & FCC & 0.00 & 3.38 & -0.22 \\
TOP-FCC & TOP & 0.33 & 3.14 & -0.21 \\
TOP-HCP & HCP & 0.17 & 3.37 & -0.22 \\
TOP-HCP & TOP & 0.45 & 3.15 & -0.22 \\
HCP-FCC & FCC & 0.64 & 3.35 & -0.21 \\
HCP-FCC & HCP & 0.66 & 3.36 & -0.21 \\
\hline
\end{tabular}

Electronic properties of graphene doped with substitutional Mn. Our DFT calculations yield significantly different energies for substitutional Mn in different stacking regions and sites (Table 1). For the most stable TOP-FCC stacking the two possible sites, namely FCC and TOP, differ for $0.33 \mathrm{eV}$. A similar value is also found for the two substitutional configurations of the TOP-HCP stacking while the site dependence is less marked in the HCP-FCC case. On the other hand taking the same site for different stacking we found energy differences ranging between 0.12 (TOP-site) and $0.64 \mathrm{eV}$ (FCC-site). On the basis 
of these results we can conclude that the substitution in TOP position is unfavoured while the FCC site is preferred and its stability is dependent on the local stacking. We were able to clearly identify the stacking-site configuration (Figure 2(e)) for only a few substitutional defects, since it requires a clear imaging of the defect as well as of the Moiré superstructure (e.g. Figure 2(e)). Although the observed trend is consistent with the calculated total energies, i.e. predominance of the two lowest energy configurations (TOP-FCC stacking with Mn in the FCC site and TOP-HCP stacking, with Mn in the HCP site), the present statistics is too low to be conclusive. Future studies should investigate these effects in more detail. Nevertheless, our DFT calculations yield similar electronic properties for all the six simulated stacking-site configurations of substitutional Mn. The Mn atom is characterized by a reduction of electrons $(-0.33$ e) with respect to the ideal atomic valence. The $n$-doping of the graphene layer $(+0.62$ e) is slightly smaller than in the bare vacancy case $(+0.75$ e). The electrons donated by the Mn atom provide about half of the charge that is necessary for charge stability of the graphene+Mn overlayer, resulting in less charge donated by the substrate $(-0.30$ e) compared to the bare vacancy case $(-0.75$ e). The charge transfer also influences the magnetic properties of the system (Table 1), in particular in the close surrounding of the substitutional atom. We obtain calculated magnetic moments for Mn $\left(\mu_{\mathrm{Mn}}\right)$ around $3.4 \mu_{B}$, and for the $\mathrm{C}$ nearest neighbours $\left(\mu_{\mathrm{C}}\right)$ around $0.2 \mu_{B}$, i.e. lower in absolute value compared to the bare vacancy case $\left(0.49 \mu_{B}\right)$. Moreover, $\mu_{\mathrm{C}}$ is anti-parallel with respect to $\mu_{\mathrm{Mn}}$ (the reason for the negative values of $\mu_{\mathrm{C}}$ in Table 1).

While so far we discussed essentially local electronic properties, we now turn our attention to the effect on Mn doping on the overall electronic properties (band structure) of graphene. Our DFT calculations yield a magnetic ground state for substitutional $\mathrm{Mn}$ in graphene/ $\mathrm{Cu}$, as can be deduced from the density of states (Figure 4(a, b)) projected on different species (PDOS). In addition to the spin-polarized PDOS of Mn we find a $0.15 \mathrm{eV}$ shift between majority and minority spin components at the Dirac cone of graphene (Figure 4(a, b) and Figure S10), confirming the interaction between the Mn atom and the carbon overlayer. In 

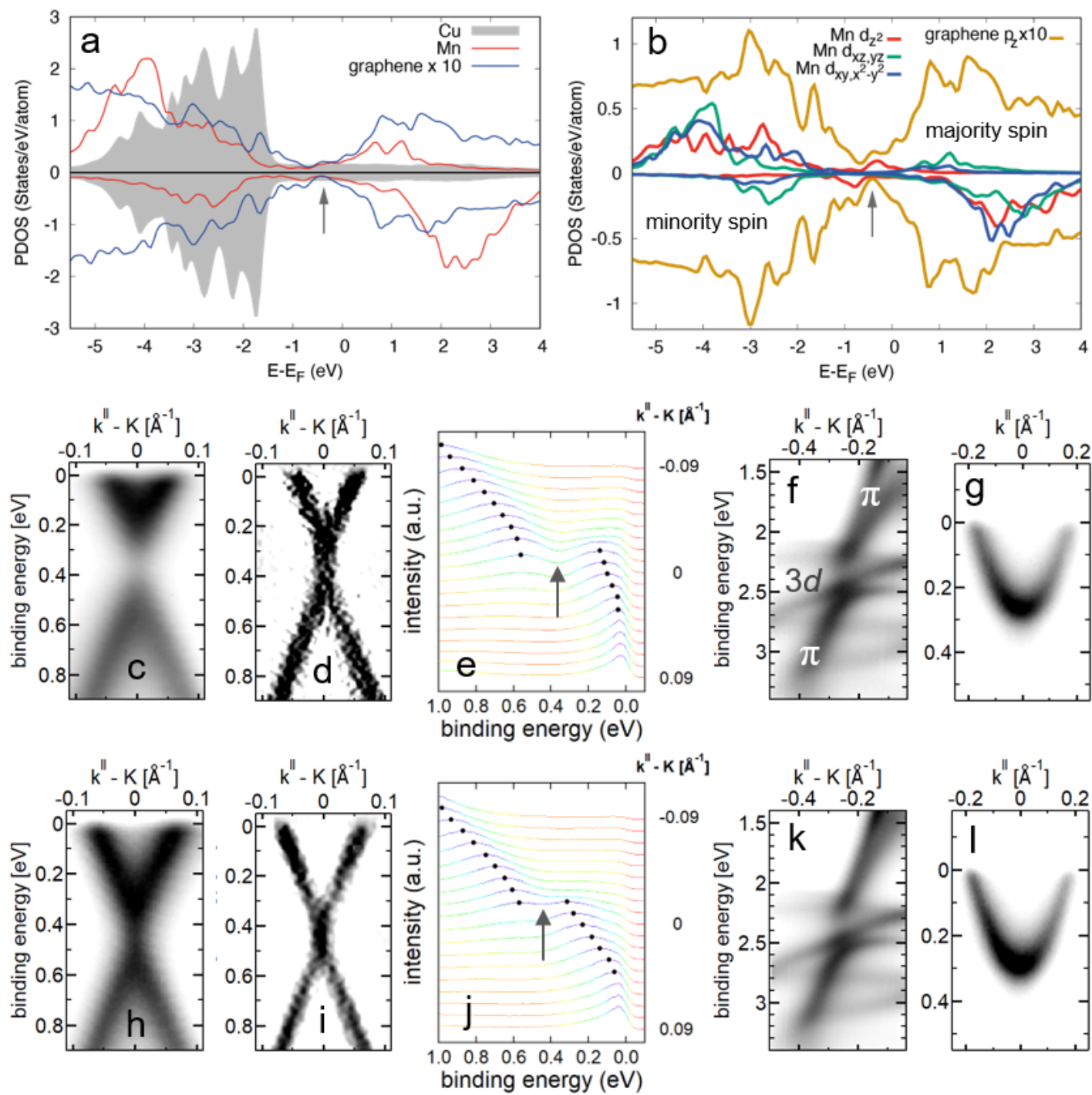

Figure 4: $(\mathrm{a}, \mathrm{b})$ Density of states (PDOS) projected on the different elements (a) as well as on the different orbital components (i.e. magnetic quantum number $m$ ) of $\mathrm{Mn}$ and $p_{z}$ states of graphene (b), for a substitutional Mn concentration of $1 \%$. The majority and minority spin components are plotted as positive and negative PDOS, respectively. (c-l) ARPES spectra $(\mathrm{h} \nu=34 \mathrm{eV})$ measured after $700{ }^{\circ} \mathrm{C}$ annealing, on pristine $(\mathrm{c}-\mathrm{g})$ and $\mathrm{Mn}$ implanted (h-l) samples. Panels (c, h) show 2D spectra measured near the K-point along the $\Gamma \rightarrow \mathrm{K}$ direction; panels $(\mathrm{d}, \mathrm{i})$ display the second derivative of the data in $(\mathrm{c}, \mathrm{h})$, highlighting the Dirac cone dispersion of the $\pi$ and $\pi^{*}$ bands of graphene. (e, j) Energy distribution curves (EDC) extracted from the p-polarization branch of the data in $(\mathrm{c}, \mathrm{h})$, with a wavenumber $(k)$ step of $0.01 \AA^{-1}$. (f, k) 2D spectra measured in the region where the graphene $\pi$-band and the $\mathrm{Cu} 3 d$-band intersect; the observed discontinuity in the graphene $\pi$-band results from hybridization with the $\mathrm{Cu} 3 d$-band. (g,l) $\mathrm{Cu}(111)$ surface state measured at the $\Gamma$ point. In panels $(e, j)$, the dots mark the local maxima. The arrows in panels $(a, b, e, j)$ point to the gap region, indicating that $\mathrm{Mn} d_{z}$ states emerge in $\mathrm{j}$ according to the PDOS in $\mathrm{a}$, $\mathrm{b}$. In the ARPES panels (c-l), binding energy is defined as $B E=E_{F}-E$. 
particular, the PDOS resolved in orbital components shows the hybridization between $d_{z^{2}}$ orbitals of Mn and $p_{z}$ states of graphene at the Fermi level while the states belonging to the other $d$ components lie far from the Dirac cone. The PDOS of the substrate is not spinpolarized and no changes are visible in the $d$-band with respect to the clean surface, if the k-integrated PDOS is considered. On the other hand, the PDOS at $\bar{\Gamma}$ (not shown) exhibits a shift of the Shockley state of $\mathrm{Cu}$ toward lower energies with respect to graphene with a bare carbon vacancy on the same substrate, in agreement with the smaller charge transfer from the substrate to the overlayer, as discussed above. Additional details on the calculated band structure are given in supplementary information (Figure S9 and Figure S10).

Figure 4(c-l) compares ARPES data for pristine (c-g) and Mn-implanted graphene (h-l), both after annealing at $700{ }^{\circ} \mathrm{C}$. The first observation is that the data (measured at $80 \mathrm{~K}$ ) do not provide clear indication of the spin-splitting predicted by DFT (corresponding to an effective temperature of $0 \mathrm{~K}$ ), i.e. a single set of Dirac cones is observed, without indication of $0.15 \mathrm{eV}$ spin-splitting predicted by DFT (Figure S10). The DOS near the Dirac point does increase as predicted for substitutional Mn ( $c f$. arrows in Figure 4, panels a, b, e, j), but finer details cannot be resolved. In fact, the ARPES measurements (Figure 4) show only a subtle effect of Mn doping in the band structure. We observe an increase in the $n$-doped character of the implanted sample (Figure $4(\mathrm{c}, \mathrm{d})$ and $(\mathrm{h}, \mathrm{i})$ ): the Fermi level position with respect to the Dirac point changes from $0.33(1) \mathrm{eV}$ in the pristine sample to $0.43(1) \mathrm{eV}$ in the implanted sample. A decrease in the band gap is also observed, from $0.40(2) \mathrm{eV}$ to $0.23(2) \mathrm{eV}$. Other key features are essentially unaffected: the $\mathrm{Cu}$ surface state ( $\mathrm{g}$ and $\mathrm{l}$ ) is clearly visible, indicating that no significant oxidation of the interface has occurred $;^{28}$ the hybridization between $\mathrm{Cu} 3 d$ and graphene $\pi$ states ( $\mathrm{f}$ and $\mathrm{k}$ ) is also clearly visible, indicating that the $\mathrm{Cu}$-graphene coupling is not significantly affected; no change in Fermi velocity can be resolved $\left(0.91(3) \times 10^{6} \mathrm{~ms}^{-1}\right.$ and $0.88(4) \times 10^{6} \mathrm{~ms}^{-1}$ for pristine and implanted, respectively). The observed $n$-doping effect in the pristine sample is well-understood as due to charge transfer from the $\mathrm{Cu}$ surface, ${ }^{29}$ the increase upon Mn implantation and annealing is unlikely 
to be a result of substitutional Mn doping, but instead an effect of the intercalated Mn component, for the following two reasons. First, our DFT calculations for substitutionally doped graphene show no increase of $n$-doping effect, which is instead found for intercalated Mn (Figure S9). Second, after $700{ }^{\circ} \mathrm{C}$ annealing, our STM data shows intercalated objects which are likely to correspond to intercalated Mn islands (Figure S3(e)). Based on the understanding that charge transfer effects in graphene-metal interface are mostly dependent on the work functions, ${ }^{29}$ and considering that the work function of $\mathrm{Mn}[4.1(2) \mathrm{eV}]$ is lower than that of $\mathrm{Cu}[4.65(5) \mathrm{eV}],{ }^{30}$ we can therefore expect a measurable $n$-doping effect from the Mn islands. Moreover, if intercalated Mn (as isolated Mn atoms) are still present after $700{ }^{\circ} \mathrm{C}$ (i.e. have not segregated into islands and have not diffused in the the $\mathrm{Cu}$ layer), they are also expected to contribute to $n$-doping on the basis of our DFT calculations. The band gap in the pristine sample is a well-known effect for graphene on $\mathrm{Cu}(111)^{31,32}$ (resulting from the hybridization between $\mathrm{Cu} 3 d$ and graphene $\pi$ states), which is overestimated due to directional broadening when the ARPES beam probes multiple grains which are not perfectly aligned with respect to each other, ${ }^{31}$ as is the present case. The apparent decrease in band gap in the Mn-doped sample is likely due to Mn DOS (mostly $d_{z^{2}}$ orbitals) emerging near the Dirac point ( $c f$. arrows in Figure $4(\mathrm{a}, \mathrm{b}, \mathrm{e}, \mathrm{j})$, and hybridizing with the $p_{z}$ states of graphene, as predicted by our DFT calculations (Figure 4 (a, b)). Indeed, in the Mn-doped case, while an energy gap can still be extracted by taking the energy difference between local DOS maxima, (arrow in Figure $4(\mathrm{j})$ ), the DOS is nearly constant in that region, i.e. there is effectively no gap. In short, the Dirac-like character of graphene is preserved upon substitutional Mn doping, with strong indication of hybridization between Mn and graphene states near the Dirac point. This makes Mn-doped graphene an ideal system in which to study strong coupling between local magnetic moments and Dirac electrons, as we discuss below in the context of future research. 
Substitutional Mn formation and stability. There are essentially two possible paths for the formation of substitutional $\mathrm{Mn}$ in graphene by ULE ion implantation: (1) direct substitution in which the impinging $\mathrm{Mn}$ ion displaces a $\mathrm{C}$ atom and takes its position; (2) a diffusion-assisted mechanism in which an implanted Mn atom (adatom or intercalated) diffuses (in plane) and becomes trapped in a $\mathrm{C}$ vacancy which had been created by an arbitrary $\mathrm{Mn}$ ion. Both mechanisms are likely to take place in the present case and both require the formation of a $\mathrm{C}$ vacancy, which by simple kinematics (conservation of kinetic energy and momentum) ${ }^{33}$ requires a minimum initial energy of the impinging ion given by:

$$
E_{\text {min }}=E_{t} \frac{\left(M_{\mathrm{Mn}}+M_{\mathrm{C}}\right)^{2}}{4 M_{\mathrm{Mn}} M_{\mathrm{C}}}
$$

where $E_{t}$ is the $\mathrm{C}$ threshold displacement energy (i.e. minimum energy that must be transferred to the $\mathrm{C}$ atom to create a stable $\mathrm{C}$ vacancy), and $M_{\mathrm{Mn}}$ and $M_{\mathrm{C}}$ are the atomic mass of Mn and $\mathrm{C}$, respectively. With $E_{t} \approx 22 \mathrm{eV},{ }^{34}$ equation 1 gives $E_{\min } \approx 37 \mathrm{eV}$. This minimum energy corresponds to a head-on collision (zero impact parameter). The larger the impact parameter, the smaller the transferred energy becomes, i.e. Mn atoms impinging with larger impact parameter with respect to the closest $\mathrm{C}$ atom must have an energy higher than 37 $\mathrm{eV}$ in order to still displace a $\mathrm{C}$ atom. We chose an implantation energy of $40 \mathrm{eV}$, i.e. above the threshold of $37 \mathrm{eV}$, so that a non-vanishing fraction of the implanted Mn ions transfer sufficient energy to create a vacancy, but not too high, to minimize the Mn backscattering energy (leading to loss of Mn to the vacuum).

From our DFT calculation we can also evaluate the stability of substitutional Mn. The binding energy between an implanted Mn atom and a vacancy can be estimated as:

$$
E_{\mathrm{b}}=\left(E_{\mathrm{Mn}-\mathrm{sub}}-E_{\mathrm{gr} @ \mathrm{Cu}}\right)-\left(E_{\mathrm{Vac}-\mathrm{gr} @ \mathrm{Cu}}-E_{\mathrm{gr} @ \mathrm{Cu}}\right)-\left(E_{\mathrm{Mn}-\text { interc }}-E_{\mathrm{gr} @ \mathrm{Cu}}\right)=-5.3 \mathrm{eV}
$$

where $E_{\mathrm{Mn}-\text { sub }}$ and $E_{\mathrm{Mn}-\text { interc }}$ are the total energies of the system with $\mathrm{Mn}$ in substitutional and intercalated positions (i.e. with and without an associated carbon vacancy), $E_{\text {gr@Cu }}$ 
is the total energy of pristine graphene on $\mathrm{Cu}(111)$, and $E_{\mathrm{Vac}-\mathrm{gr} @ \mathrm{Cu}}$ of that system with a single vacancy. Such a strong binding $(-5.3 \mathrm{eV})$ confirms the tendency of the implanted Mn atoms to combine with existing vacancies in the graphene lattice, as well as the high thermal stability of the substitutional configuration (at least up to $700{ }^{\circ} \mathrm{C}$, as reported here).

While in the present case (ULE ion implantation), both direct and diffusion-assisted substitution is likely to occur, two-step doping approaches in which the vacancy formation and the incorporation of transition metals (e.g. Fe, Co, Ni, Mo, Pt) correspond to two separate processes ${ }^{11,12,18,35}$ are essentially based on the diffusion-assisted mechanism. For example, vacancies are formed by electron irradiation, followed by thermal deposition of the metal atoms, which diffuse on the surface until becoming trapped in the previously formed vacancies. Diffusion-assisted substitution has been observed to be thermally activated (i.e. occurring only upon annealing above room temperature); ${ }^{35}$ this would imply that, if part of substitutional Mn in our samples was indeed formed via a diffusion assisted-mechanism (i.e. not exclusively by direct substitution), the post-implantation annealing played a critical role. Since its atomic number is similar to $\operatorname{Mn}(Z=25)$, it is interesting to further compare our results (in terms of defect structure, electronic properties and stability) to those previously obtained for $\mathrm{Fe}(Z=26)$ doping via two-step processes. The atomic structures of the two types of defects are similar (Mn here and Fe in Ref. 12, in a single C vacancy), characterized by strong relaxation effects resulting from the large effective radius of $\mathrm{Mn} / \mathrm{Fe}$ compared to the $\mathrm{C}$ atom that they substitute: in-plane displacement of the neighboring $\mathrm{C}$ atoms away from the metal atom; out-of-plane displacement of the metal atom and (although less pronounced) of the neighboring $\mathrm{C}$ atoms. There is, however, a difference in the latter (outof-plane displacement), already mentioned above, resulting from the fact that in the present work the graphene layer is supported on a substrate while in Ref. 12 it is suspended. While in suspended graphene, it is generally accepted that the out-of-plane displacement of the metal dopant and of neighboring $\mathrm{C}$ atoms occurs in the same direction, in our case, as mentioned above, the $\mathrm{Cu}$ substrate breaks the mirror symmetry with respect to the graphene plane. 
Consequently, the metal dopant $(\mathrm{Mn})$ is observed to displace towards the $\mathrm{Cu}$ surface whereas the neighboring $\mathrm{C}$ atoms are displaced away from it, resulting in a total energy that is 2.25 $\mathrm{eV}$ lower than for a configuration in which both the $\mathrm{Mn}$ and $\mathrm{C}$ atoms are displaced away from the $\mathrm{Cu}$ surface. In terms of electronic structure there is a striking difference between substitutional $\mathrm{Mn}$ and Fe: while for Mn the electrons in (different) non-bonding $d$ orbitals add up to a local moment of approximately $3 \mu_{B}$, in Fe the $3 d$ spins pair up resulting in a vanishing local moment. ${ }^{26}$ In terms of stability, the fact that we observe no signs of instability up to $700{ }^{\circ} \mathrm{C}$ supports the calculated high binding energies of metal atoms such as Mn and Fe in single $\mathrm{C}$ vacancies: $\sim-5 \mathrm{eV}$ for substitutional $\mathrm{Mn}$ in graphene $/ \mathrm{Cu}(111)$ as reported here, and $\sim-6 \mathrm{eV}$ and $\sim-7 \mathrm{eV}$ for $\mathrm{Mn}$ and Fe, respectively, in suspended graphene. ${ }^{26}$ The fact that we do not observe signs of clustering of substitutional Mn atoms up to $700{ }^{\circ} \mathrm{C}$ is also consistent with calculated migration barriers of $\sim 3 \mathrm{eV}$ for $3 d$ metals. ${ }^{26}$ As mentioned in Ref. 35, the lower stability observed in that work is likely a consequence of a different defect structure, i.e. not an ideal substitution into a single $\mathrm{C}$ vacancy.

Future studies should evaluate the roles of direct and diffusion-assisted substitution as well as the effect of thermal annealing and varying ion energy on the substitution efficiency, in particular, towards higher substitutional concentrations. In the present work, with an implantation energy of $40 \mathrm{eV}$, the $0.04 \%$ substitutional concentration (determined from STM) corresponds to about $1 \%$ efficiency with respect to the implanted fluence. Further optimization, in particular towards slightly higher energies, is likely to further increase the substitutional efficiency, by increasing the vacancy formation rate, without significantly increasing the Mn backscattering energy. In any case, the substitutional concentration can in principle be increased by increasing the fluence. For example, a substitutional concentration of about $1 \%$ (with a substitution efficiency of a few \%) was obtained with ULE ion implantation $\left(25 \mathrm{eV}\right.$ ) of Nitrogen ${ }^{20}$ (for $\mathrm{N}$, equation 1 gives $E_{\min } \approx 22 \mathrm{eV}$, i.e. also slightly below the implantation energy, as in the present case). 
Prospects for magnetic functionality. As mentioned above, ARPES measurements at $80 \mathrm{~K}$ do not provide evidence of the spin-splitting (ferromagnetic state) predicted by DFT (corresponding to an effective temperature of $0 \mathrm{~K}$ ). Various reasons may explain this. First, the ordering temperature may simply be below $80 \mathrm{~K}$. Second, the local magnetic moment of substitutional Mn may in fact be strongly decreased or completely screened by the conduction electrons (Kondo effect, which has been reported for magnetic defects in graphene at temperatures as high as $90 \mathrm{~K}^{36}$ ), which would inhibit a ferromagnetic state. Future studies should therefore specifically address the magnetic properties of substitutionally doped graphene. On the theoretical side, more complex approaches including many-body effects related to electronic correlation can be adopted, such as tight binding model with Hubbard term ${ }^{37}$ or DFT+dynamical mean-field theory (DMFT) in the Anderson Impurity Model ${ }^{38}$ which allow to describe Kondo physics. On the experimental side, various advanced techniques can be applied that provide insight at various scales, for example: local studies based on scanning tunneling microscopy and spectroscopy, including with spin-polarized capabilities; ${ }^{3,8,39}$ macroscopic studies based on X-ray magnetic circular dichroism (XMCD); ${ }^{40,41}$ spin-resolved band structure characterization based on spin-resolved ARPES; ${ }^{42}$ magnetotransport studies (e.g. Hall effect and magnetoresistance measurements). ${ }^{36}$

Magnetic doping of 2D materials by ULE ion implantation. Our approach to magnetic substitutional doping, based on ULE ion implantation, offers various advantages. For example, contrary to two-step approaches, ${ }^{11,12,18}$ we can control both processes of vacancy creation and of dopant incorporation with a single set of implantation parameters, which can be expected to yield better reproducibility. All the implantation parameters (energy, fluence, fluence rate, and temperature) can be precisely monitored and controlled, which in general provide high degree of control and reliability. Moreover, the resulting atomic configuration, in which the Mn atom is underneath (protected by) the graphene layer, can be expected to provide more stability with respect to exposure to the atmosphere and post-processing 
steps (e.g. deposition of a polymer layer for transfer or of an oxide layer for top gating). The approach is also extremely flexible, being compatible with virtually any element of the periodic table (as dopant) and any 2D material (as host). Although one can expect some limitations regarding which elements can effectively be substitutionally incorporated into a given 2D material, those should be less restrictive compared to processes that occur closer to equilibrium (e.g. doping during growth). Therefore, the present work motivates further research, even beyond magnetic doping of graphene, for example: magnetic doping of $\mathrm{MoS}_{2}$ and related 2D semiconductors for spintronics and valleytronics applications; ${ }^{43}$ creation of spin defects in h-BN and related $2 \mathrm{D}$ insulators for quantum technologies. ${ }^{44,45}$ Finally, ion implantation is widely used for semiconductor processing in microelectronics; if ULE ion implantation technology is further developed to the level of its higher energy counterpart, one can expect it to be compatible with wafer-scale fabrication.

\section{Conclusion}

To conclude, we demonstrate the incorporation of substitutional Mn in graphene using ULE ion implantation and provide a detailed characterization of its local atomic structure. We also show that graphene doped with substitutional Mn retains the Dirac-like behavior of pristine graphene, making it an ideal system in which to study the electronic and magnetic interplay between local magnetic moments and Dirac electrons. These results establish a solid basis for such future studies, for example, dealing with ferromagnetism and Kondo physics in magnetically doped 2D materials. Although this work deals with Mn doping of graphene, our approach is compatible with virtually any dopant element and any 2D material host. 


\section{Methods}

CVD growth of graphene. Pre-cut and polished 2" Czochralski grown sapphire wafers (Roditi International Corporation) diced along the c-plane are used to prepare $\mathrm{Al}_{2} \mathrm{O}_{3}$ template wafers. The monocrystalline wafers have a surface misorientation smaller than $0.3^{\circ}$. The wafers were cleaned prior to $\mathrm{Cu}$ sputtering in a $3: 1$ concentrated acid mixture of $\mathrm{H}_{2} \mathrm{O}_{4}: \mathrm{H}_{3} \mathrm{PO}_{4}$ at $200{ }^{\circ} \mathrm{C}$ for $20 \mathrm{~min}$ followed by a UPW rinse for 3 min. Cleaned sapphire wafers are placed in a Nimbus 310 sputtering setup with a base pressure of $4 \times 10^{-6}$ mbar. Substrates are placed using a $200 \mathrm{~mm}$ Si pocket wafer and sputtering is performed at room temperature for $173 \mathrm{~s}$ (21 passes under target) at $6 \times 10^{-3}$ mbar Ar pressure. The $\mathrm{Cu}$ deposition rate is calibrated and corresponds to a rate of about $29 \AA \mathrm{s}^{-1}$. The applied power was $3000 \mathrm{~W}$ and the throw distance is approximately $50 \mathrm{~mm}$. The graphene growth process has been optimized taking into account oriented monolayer growth and low Raman D peak. The growth conditions are $5000 \mathrm{sccm}$ Ar: $125 \mathrm{sccm} \mathrm{H}_{2}: 0.3 \mathrm{sccm} \mathrm{CH}_{4}$ at a pressure of 750 mbar close to the melting point of $\mathrm{Cu}$. The growth time is $30 \mathrm{~min}$, while ramp-up and down is performed in a mixture of $\mathrm{Ar}$ and $\mathrm{H}_{2}$.

Scanning tunneling microscopy. The STM micrographs reported here were obtained in UHV (base pressure $\sim 10^{-11}$ mbar) at low temperatures $(2 \mathrm{~K}, 5 \mathrm{~K}$ and $78 \mathrm{~K}$ ) using an Omicron LT STM and an Unisoku USM-1500. We used electrochemically etched W tips and cut Pt-Ir tips. The tip oxide from the $\mathrm{W}$ tips is first removed by flash annealing. Both types of tips were characterized by scanning the $\mathrm{Au}(111)$ surface. All topographies were acquired in constant $I_{\text {tun. }}$.

X-ray photoelectron spectroscopy. XPS measurements were performed at the SuperESCA beamline at Elettra synchrotron in Trieste. All the spectra were acquired in UHV (base pressure $\sim 10^{-10}$ mbar) and at room temperature (RT), the samples were placed in the normal emission (NE) configuration, with respect to the hemispherical analyzer. The 
samples were annealed in-situ up to $700{ }^{\circ} \mathrm{C}$, with a ramp rate of $1{ }^{\circ} \mathrm{C} / \mathrm{sec}$ to monitor the change of Mn concentration. The C 1s, O 1s, and Mn 2p core-level spectra were acquired at photon energies of $400 \mathrm{eV}, 650 \mathrm{eV}$, and $780 \mathrm{eV}$, respectively. The core-level spectra were fitted with Doniach-Šunjić functions convoluted with a Gaussian distribution and a linear background (apart from Mn 2p core-level, where a Shirley background is used).

Raman spectroscopy. Raman spectra were measured using a confocal Raman microscope (Monovista CRS+, S\&I GmbH) equipped with a $532 \mathrm{~nm} \mathrm{Nd:YAG} \mathrm{laser.} \mathrm{The} \mathrm{laser} \mathrm{was}$ directed onto the sample surface through an objective (OLYMPUS, BX43 100×, N.A. 0.7), with the maximum laser power remaining below $1 \mathrm{~mW}$ in order to avoid laser-induced modification. All the measurements were obtained in ambient conditions, at RT.

Angle-resolved photoemission spectroscopy. ARPES measurements were performed at the BaDElPh beamline at Elettra synchrotron in Trieste, ${ }^{46}$ with a photon energy of 34 $\mathrm{eV}$. Before the measurements, the sample was pre-annealed at $700{ }^{\circ} \mathrm{C}$. The ARPES spectra were obtained in UHV (base pressure $\sim 5 \times 10^{-11} \mathrm{mbar}$ ), at $80 \mathrm{~K}$. The presented spectra correspond to a summation of data that were separately acquired using linear p- and spolarized light. The energy and angular resolution were set to $10 \mathrm{meV}$ and $0.1^{\circ}$, respectively.

Low-energy electron diffraction. As-grown graphene samples were characterized using low-energy electron diffraction (LEED) measured in an UHV chamber with background pressure better than $4 \times 10^{-9} \mathrm{mbar}$ using a rear-view AEP 8011, VG Scientific instrument. LEED images were recorded using the screen voltage and an electron beam (spot size ca. $1 \mathrm{~mm})$ energy of $1 \mathrm{kV}$ and $52 \mathrm{eV}$, respectively.

Density functional theory calculations. We performed Density Functional Theory calculations with generalized gradient approximation (GGA) for the exchange-correlation potential, using a localized orbital basis set and a pseudopotential description of the core elec- 
trons, as implemented in the SIESTA code. In the adopted model graphene is matched to the underlying $\mathrm{Cu}(111)$ surface, by taking the theoretical lattice constant $\left(a_{\text {Theo }}=3.59 \AA\right)$, resulting in a $3 \%$ expansion of the overlayer. We considered one defect related to Mnimplantation in a $(7 \times 7)$ supercell, corresponding to having a Mn-C ratio of $1 / 97 \sim 1 \%$. Instead of dealing with Moiré reconstruction that would require a very large periodicity, we separately analyze different the possible stacking configurations, corresponding to different high-symmetry regions of the Moiré, placing the Mn atom in different positions with respect to the graphene lattice and the underlying substrate. This choice is supported by the local nature of the graphene- $\mathrm{Mn}-\mathrm{Cu}$ interaction in proximity of the defect, leading to electronic, magnetic and structural properties that are not dependent on the long range arrangement typical of the Moiré. We performed structural relaxation of the overlayer with the Mn atom while the $\mathrm{Cu}$ is taken as fixed to the relaxed coordinate of the surface. The threshold for the force tolerance was set to $0.04 \mathrm{eV} / \AA$. We adopted a mesh cutoff of 450 Ry and a $5 \times 5 \times 1$ sampling of the Brillouin zone. Along the direction perpendicular to the surface three layers of $\mathrm{Cu}$ were considered for structural relaxation while a six layer $\mathrm{Cu}$ slab was adopted for the evaluation of the electronic properties. The periodic replica of the system along the same direction were separated by a $40 \AA$ wide vacuum region.

The STM simulations were performed in a Tersoff-Hamman approach, ${ }^{47}$ assuming a constant density of states for the tip. The integration range for the electronic density, corresponding to the applied bias, was varied in an energy interval of $1.0 \mathrm{eV}$ around the Fermi level. We simulated a constant-height (3 A ) STM image applying a Gaussian spatial broad-

ening of $1 \AA$ to the electronic density to mimic the finite experimental resolution associated with the tip.

\section{Acknowledgement}

This work was funded by KU Leuven and FWO Vlaanderen. M.N.N. acknowledges funding 
from the EU Horizon 2020 Framework (MagDirac project, ID:796940). K.V. also acknowledges funding from the Agency for Innovation \& Entrepreneurship (VLAIO). The authors acknowledge Elettra Sincrotrone Trieste for providing access to its synchrotron radiation facilities and, in particular, to the BaDEIPh and SuperESCA beamlines, as well as the Red Espanola de Supercomputacion (RES) for the availability of high performance computing resources and support under the project FI-2020-2-0034. The access to the Elettra Sincrotrone Trieste has been supported by the project CALIPSOplus under Grant Agreement 730872 from the EU Framework Programme for Research and Innovation HORIZON 2020. The authors thank P. Lacovig and S. Lizzit for the support during the XPS beam time at SuperESCA, Elettra. The authors also thank P. Walke for the support with the Raman spectroscopy measurements.

\section{Supporting Information Available}

The Supporting Information is available free of charge on the ACS Publications website at $\cdots$

It provides additional results from the DFT calculations and from the experimental studies.

\section{References}

(1) Jiang, Y.; Lo, P.-W.; May, D.; Li, G.; Guo, G.-Y.; Anders, F. B.; Taniguchi, T.; Watanabe, K.; Mao, J.; Andrei, E. Y. Inducing Kondo Screening of Vacancy Magnetic Moments in Graphene with Gating and Local Curvature. Nature Communications 2018, 9, 1-7.

(2) González-Herrero, H.; Gómez-Rodríguez, J. M.; Mallet, P.; Moaied, M.; Palacios, J. J.; Salgado, C.; Ugeda, M. M.; Veuillen, J.-Y.; Yndurain, F.; Brihuega, I. Atomic-Scale 
Control of Graphene Magnetism by Using Hydrogen Atoms. Science 2016, 352, 437441.

(3) Ren, J.; Guo, H.; Pan, J.; Zhang, Y. Y.; Wu, X.; Luo, H.-G.; Du, S.; Pantelides, S. T.; Gao, H.-J. Kondo Effect of Cobalt Adatoms on a Graphene Monolayer Controlled by Substrate-Induced Ripples. Nano Letters 2014, 14, 4011-4015.

(4) Baltic, R.; Donati, F.; Singha, A.; Wäckerlin, C.; Dreiser, J.; Delley, B.; Pivetta, M.; Rusponi, S.; Brune, H. Magnetic Properties of Single Rare-Earth Atoms on Graphene/Ir (111). Physical Review B 2018, 98, 024412.

(5) Ajejas, F.; Gudín, A.; Guerrero, R.; Anadón Barcelona, A.; Diez, J. M.; de Melo Costa, L.; Olleros, P.; Niño, M. A.; Pizzini, S.; Vogel, J.; Valvidares, M.; Gargiani, P.; Cabero, M.; Camarero, J.; Miranda, R.; Perna, P. Unraveling DzyaloshinskiiMoriya Interaction and Chiral Nature of Graphene/Cobalt Interface. Nano Letters 2018, 18, 5364-5372.

(6) Candini, A.; Klyatskaya, S.; Ruben, M.; Wernsdorfer, W.; Affronte, M. Graphene Spintronic Devices with Molecular Nanomagnets. Nano letters 2011, 11, 2634-2639.

(7) Babar, R.; Kabir, M. Ferromagnetism in Nitrogen-Doped Graphene. Physical Review B 2019, 99, 115442.

(8) Ren, J.; Guo, H.; Pan, J.; Zhang, Y.-F.; Yang, Y.; Wu, X.; Du, S.; Ouyang, M.; Gao, H.-J. Interatomic Spin Coupling in Manganese Clusters Registered on Graphene. Phys. Rev. Lett. 2017, 119, 176806.

(9) del Castillo, E.; Cargnoni, F.; Achilli, S.; Tantardini, G.; Trioni, M. Spin Symmetric Band Gap Opening in Graphene by Fe Adsorption. Surf. Sci. 2015, 634, 62-67.

(10) del Castillo, E.; Achilli, S.; Cargnoni, F.; Ceresoli, D.; Soave, R.; Trioni, M. Spin- 
Filtering in Graphene Junctions with Ti and Co Adsorbates. Chem. Phys. 2016, 478, 91.

(11) Wang, H.; Wang, Q.; Cheng, Y.; Li, K.; Yao, Y.; Zhang, Q.; Dong, C.; Wang, P.; Schwingenschlogl, U.; Yang, W.; Zhang, X. Doping Monolayer Graphene with Single Atom Substitutions. Nano letters 2012, 12, 141-144.

(12) Robertson, A. W.; Montanari, B.; He, K.; Kim, J.; Allen, C. S.; Wu, Y. A.; Olivier, J.; Neethling, J.; Harrison, N.; Kirkland, A. I.; Warner, J. H. Dynamics of Single Fe Atoms in Graphene Vacancies. Nano Letters 2013, 13, 1468-1475.

(13) Zhao, L.; He, R.; Rim, K. T.; Schiros, T.; Kim, K. S.; Zhou, H.; Gutiérrez, C.; Chockalingam, S.; Arguello, C. J.; Pálová, L.; Nordlund, D.; Hybertsen, M. S.; Reichman, D. R.; Heinz, T. F.; Kim, P.; Pinczuk, A.; Flynn, G. W.; Pasupathy, A. N. Visualizing Individual Nitrogen Dopants in Monolayer Graphene. Science 2011, 333, 999-1003.

(14) Terrones, H.; Lv, R.; Terrones, M.; Dresselhaus, M. S. The Role of Defects and Doping in 2D Graphene Sheets and 1D Nanoribbons. Reports on Progress in Physics 2012, 75, 062501.

(15) Zhou, W.; Kapetanakis, M. D.; Prange, M. P.; Pantelides, S. T.; Pennycook, S. J.; Idrobo, J.-C. Direct Determination of the Chemical Bonding of Individual Impurities in Graphene. Physical Review Letters 2012, 109, 206803.

(16) Ramasse, Q. M.; Seabourne, C. R.; Kepaptsoglou, D.-M.; Zan, R.; Bangert, U.; Scott, A. J. Probing the Bonding and Electronic Structure of Single Atom Dopants in Graphene with Electron Energy Loss Spectroscopy. Nano Letters 2013, 13, 49894995.

(17) Susi, T.; Meyer, J. C.; Kotakoski, J. Manipulating Low-Dimensional Materials down 
to the Level of Single Atoms with Electron Irradiation. Ultramicroscopy 2017, 180, $163-172$.

(18) Dyck, O.; Zhang, L.; Yoon, M.; Swett, J. L.; Hensley, D.; Zhang, C.; Rack, P. D.; Fowlkes, J. D.; Lupini, A. R.; Jesse, S. Doping Transition-Metal Atoms in Graphene for Atomic-Scale Tailoring of Electronic, Magnetic, and Quantum Topological Properties. Carbon 2020, DOI:10.1016/j.carbon.2020.11.015.

(19) Bangert, U.; Pierce, W.; Kepaptsoglou, D.; Ramasse, Q.; Zan, R.; Gass, M.; Van den Berg, J.; Boothroyd, C.; Amani, J.; Hofsässs, H. Ion Implantation of Graphene - Toward IC Compatible Technologies. Nano Letters 2013, 13, 4902-4907.

(20) Willke, P.; Amani, J.; Thakur, S.; Weikert, S.; Druga, T.; Maiti, K.; Hofsäss, H.; Wenderoth, M. Short-Range Ordering of Ion-Implanted Nitrogen Atoms in SiC-Graphene. Applied Physics Letters 2014, 105, 111605.

(21) Kepaptsoglou, D.; Hardcastle, T. P.; Seabourne, C. R.; Bangert, U.; Zan, R.; Amani, J. A.; Hofsäss, H.; Nicholls, R. J.; Brydson, R. M. D.; Scott, A. J.; Ramasse, Q. M. Electronic Structure Modification of Ion Implanted Graphene: The Spectroscopic Signatures of $p$ - and n-Type Doping. ACS Nano 2015, 9, 11398-11407.

(22) Willke, P.; Amani, J. A.; Sinterhauf, A.; Thakur, S.; Kotzott, T.; Druga, T.; Weikert, S.; Maiti, K.; Hofsäss, H.; Wenderoth, M. Doping of Graphene by Low-Energy Ion Beam Implantation: Structural, Electronic, and Transport Properties. Nano Letters 2015, $15,5110-5115$.

(23) Cress, C. D.; Schmucker, S. W.; Friedman, A. L.; Dev, P.; Culbertson, J. C.; Lyding, J. W.; Robinson, J. T. Nitrogen-Doped Graphene and Twisted Bilayer Graphene via Hyperthermal Ion Implantation with Depth Control. ACS Nano 2016, 10, 37143722 . 
(24) Susi, T.; Hardcastle, T. P.; Hofsäss, H.; Mittelberger, A.; Pennycook, T. J.; Mangler, C.; Drummond-Brydson, R.; Scott, A. J.; Meyer, J. C.; Kotakoski, J. Single-Atom Spectroscopy of Phosphorus Dopants Implanted into Graphene. 2D Materials 2017, 4, 021013.

(25) Tripathi, M.; Markevich, A.; Bottger, R.; Facsko, S.; Besley, E.; Kotakoski, J.; Susi, T. Implanting Germanium into Graphene. ACS Nano 2018, 12, 4641-4647.

(26) Krasheninnikov, A.; Lehtinen, P.; Foster, A. S.; Pyykkö, P.; Nieminen, R. M. Embedding Transition-Metal Atoms in Graphene: Structure, Bonding, and Magnetism. Physical Review Letters 2009, 102, 126807.

(27) Verguts, K.; Vermeulen, B.; Vrancken, N.; Schouteden, K.; Haesendonck, C. V.; Huyghebaert, C.; Heyns, M.; Gendt, S. D.; Brems, S. Epitaxial Al2O3(0001)/Cu(111) Template Development for CVD Graphene Growth. The Journal of Physical Chemistry C 2016, 120, 297-304.

(28) Gottardi, S.; Müller, K.; Bignardi, L.; Moreno-López, J. C.; Pham, T. A.; Ivashenko, O.; Yablonskikh, M.; Barinov, A.; Björk, J.; Rudolf, P.; Stöhr, M. Comparing Graphene Growth on $\mathrm{Cu}(111)$ versus Oxidized $\mathrm{Cu}(111)$. Nano Letters 2015, 15, 917-922, PMID: 25611528.

(29) Khomyakov, P.; Giovannetti, G.; Rusu, P.; Brocks, G. v.; Van den Brink, J.; Kelly, P. J. First-Principles Study of the Interaction and Charge Transfer between Graphene and Metals. Physical Review B 2009, 79, 195425.

(30) Drummond, T. J. Work Functions of the transition Metals and Metal Silicides; Report no. SAND99-0391J; U.S. Department of Energy: Washington, DC, 1999.

(31) Avila, J.; Razado, I.; Lorcy, S.; Fleurier, R.; Pichonat, E.; Vignaud, D.; Wallart, X.; Asensio, M. C. Exploring Electronic Structure of One-Atom Thick Polycrystalline 
Graphene Films: A Nano Angle Resolved Photoemission Study. Scientific Reports 2013, 3, 2439.

(32) Vita, H.; Böttcher, S.; Horn, K.; Voloshina, E.; Ovcharenko, R.; Kampen, T.; Thissen, A.; Dedkov, Y. S. Understanding the Origin of Band Gap Formation in Graphene on Metals: Graphene on Cu/Ir (111). Scientific Reports 2014, 4, 5704.

(33) Alford, T. L.; Feldman, L. C.; Mayer, J. W. Fundamentals of Nanoscale Film Analysis; Springer Science \& Business Media: Boston, MA, 2007.

(34) Meyer, J. C.; Eder, F.; Kurasch, S.; Skakalova, V.; Kotakoski, J.; Park, H. J.; Roth, S.; Chuvilin, A.; Eyhusen, S.; Benner, G.; Krasheninnikov, A. V.; Kaiser, U. Accurate Measurement of Electron Beam Induced Displacement Cross Sections for Single-Layer Graphene. Physical Review Letters 2012, 108, 196102.

(35) Rodriguez-Manzo, J. A.; Cretu, O.; Banhart, F. Trapping of Metal Atoms in Vacancies of Carbon Nanotubes and Graphene. ACS nano 2010, 4, 3422-3428.

(36) Chen, J.-H.; Li, L.; Cullen, W. G.; Williams, E. D.; Fuhrer, M. S. Tunable Kondo Effect in Graphene with Defects. Nature Physics 2011, 7, 535-538.

(37) Liang, L.; Costa Gira, E.; Meunier, V. Modeling the Kondo Effect of a Magnetic Atom Adsorbed on Graphene. 2D Mater. 2019, 6, 035038.

(38) Jacob, D. Towards a Full ab Initio Theory of Strong Electronic Correlations in Nanoscale Devices. J. Phys. Condens. Matter 2015, 27, 245606.

(39) Choi, T. Studies of Single Atom Magnets via Scanning Tunneling Microscopy. Journal of Magnetism and Magnetic Materials 2019, 481, 150-155.

(40) Girovsky, J.; Nowakowski, J.; Ali, M. E.; Baljozovic, M.; Rossmann, H. R.; Nijs, T.; Aeby, E. A.; Nowakowska, S.; Siewert, D.; Srivastava, G.; Wäckerlin, C.; Dreiser, J.; 
Decurtins, S.; Liu, S.-X.; Oppeneer, P. M.; Jung, T. A.; Ballav, N. Long-Range Ferrimagnetic Order in a Two-Dimensional Supramolecular Kondo Lattice. Nature Communications 2017, 8, 1-8.

(41) Joly, L.; Kappler, J.-P.; Ohresser, P.; Sainctavit, P.; Henry, Y.; Gautier, F.; Schmerber, G.; Kim, D.; Goyhenex, C.; Bulou, H.; Bengone, O.; Kavich, J.; Gambardella, P.; Scheurer, F. Kondo Screening of the Spin and Orbital Magnetic Moments of Fe Impurities in $\mathrm{Cu}$. Physical Review B 2017, 95, 041108.

(42) Mo, S.-K. Angle-Resolved Photoemission Spectroscopy for the Study of TwoDimensional Materials. Nano Convergence 2017, 4, 6.

(43) Singh, N.; Schwingenschlögl, U. A Route to Permanent Valley Polarization in Monolayer MoS2. Advanced Materials 2017, 29, 1600970.

(44) Tran, T. T.; Bray, K.; Ford, M. J.; Toth, M.; Aharonovich, I. Quantum Emission from Hexagonal Boron Nitride Monolayers. Nature Nanotechnology 2016, 11, 37-41.

(45) Castelletto, S.; Inam, F. A.; Sato, S.-i.; Boretti, A. Hexagonal Boron Nitride: A Review of the Emerging Material Platform for Single-Photon Sources and the Spin-Photon Interface. Beilstein Journal of Nanotechnology 2020, 11, 740-769.

(46) Petaccia, L.; Vilmercati, P.; Gorovikov, S.; Barnaba, M.; Bianco, A.; Cocco, D.; Masciovecchio, C.; Goldoni, A. BaD ElPh: A 4 m Normal-Incidence Monochromator Beamline at Elettra. Nuclear Instruments and Methods in Physics Research Section A: Accelerators, Spectrometers, Detectors and Associated Equipment 2009, 606, 780-784.

(47) Tersoff, J.; Hamman, D. R. Theory of the Scanning Tunneling Microscope. Phys. Rev. $B$ 1985, 31, 805 . 


\section{Supplementary information for: Doping Graphene with Substitutional Mn}

\section{STM simulations (DFT) for various defects}

As mentioned in the main text, the unambiguous identification of substitutional Mn is based not only on the agreement with DFT simulations for that configuration, but also on the lack of agreement with simulations (Figure S1) of other point defects that may also be present: free single vacancies, free double vacancies, $\mathrm{Mn}$ in double vacancies, substitutional $\mathrm{Mn}$ in the outwards-protruding configuration, and intercalated Mn (without vacancy).

\section{STM simulations (DFT) for substitutional Mn}

As mentioned in the main text, for all the six high-symmetry substitutional configurations, the STM simulations (Figure S2) show generally similar features, and are in good agreement with the shape and contrast observed in the STM measurements and identified as substitutional Mn in the inwards configuration.

\section{Quantification of substitutional Mn}

The weighted average $(0.04 \%)$ and standard deviation $(0.02 \%)$ of the concentration of substitutional $\mathrm{Mn}$ (with respect to $\mathrm{C}$ atoms) were determined from the estimated concentration in 19 micrographs from different surface areas on the same sample (Table S1). The weight was determined based on the area of each micrograph with respect to the total area of all the considered micrographs.

\section{Other forms of Mn incorporation}

Different types of defects were observed in the STM micrographs that can be attributed to other forms of Mn incorporation (other than substitutional Mn in the inwards configuration), 
a)

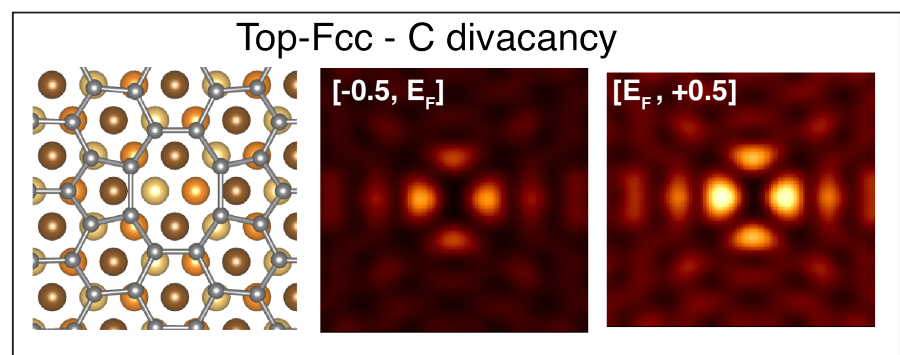

b)

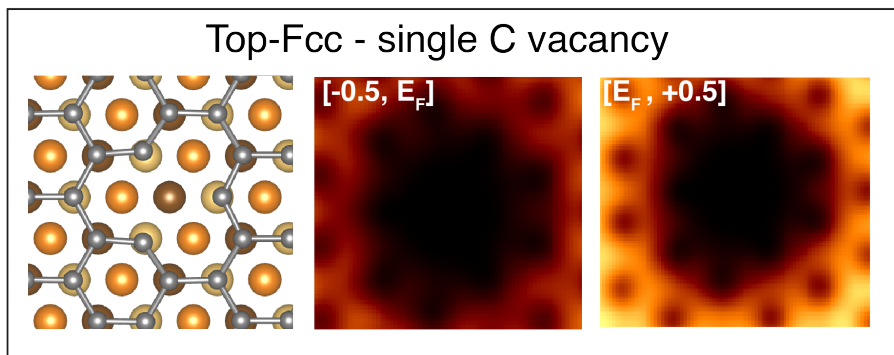

c)

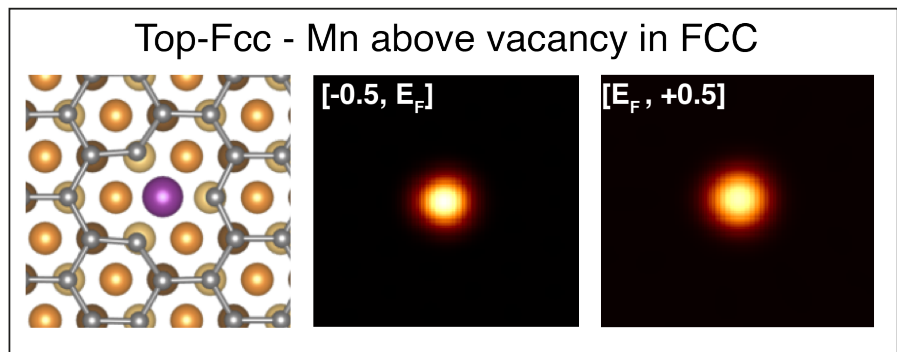

d)

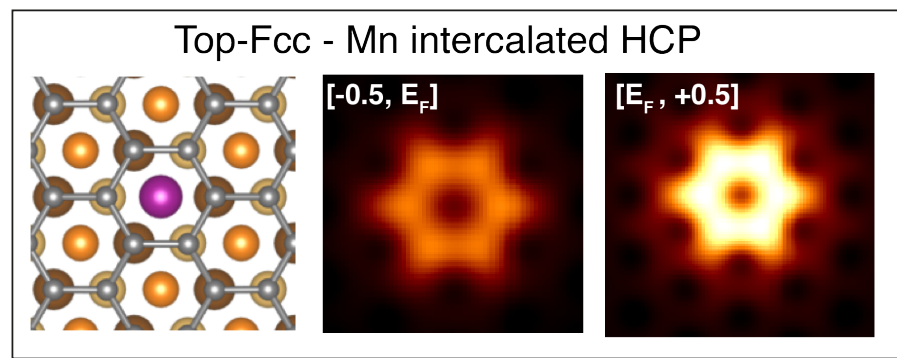

e)

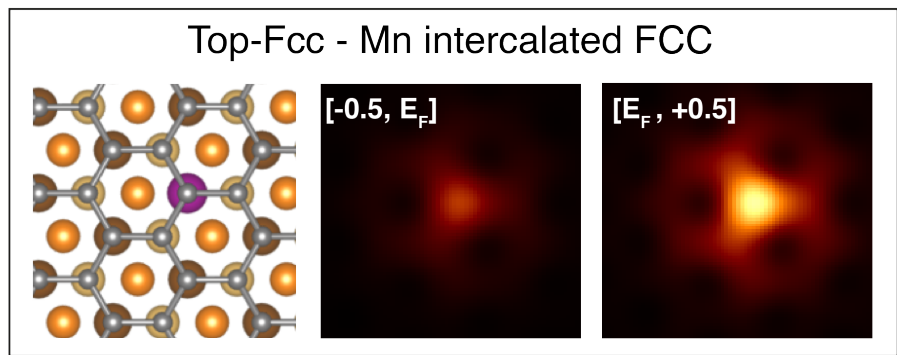

Figure S1: STM simulations for various point defects: carbon double (a) and single (b) vacancy in graphene $/ \mathrm{Cu}(111)$, substitutional $\mathrm{Mn}$ protruding outwards i.e. adsorbed on top of a $\mathrm{C}$ vacancy (c) and intercalated $\mathrm{Mn}$ in $\mathrm{HCP}(\mathrm{d})$ and $\mathrm{FCC}(\mathrm{e})$ positions in graphene/Cu(111) with the TOP-FCC stacking. All the simulations are performed with the same tip-surface distance $(3 \AA)$, with the same tip size $(1 \AA)$, and are plotted in the same contrast scale. The integration energy ranges are indicated $\left(0.5 \mathrm{eV}\right.$ below and $0.5 \mathrm{eV}$ above the Fermi level $\left.E_{F}\right)$. 

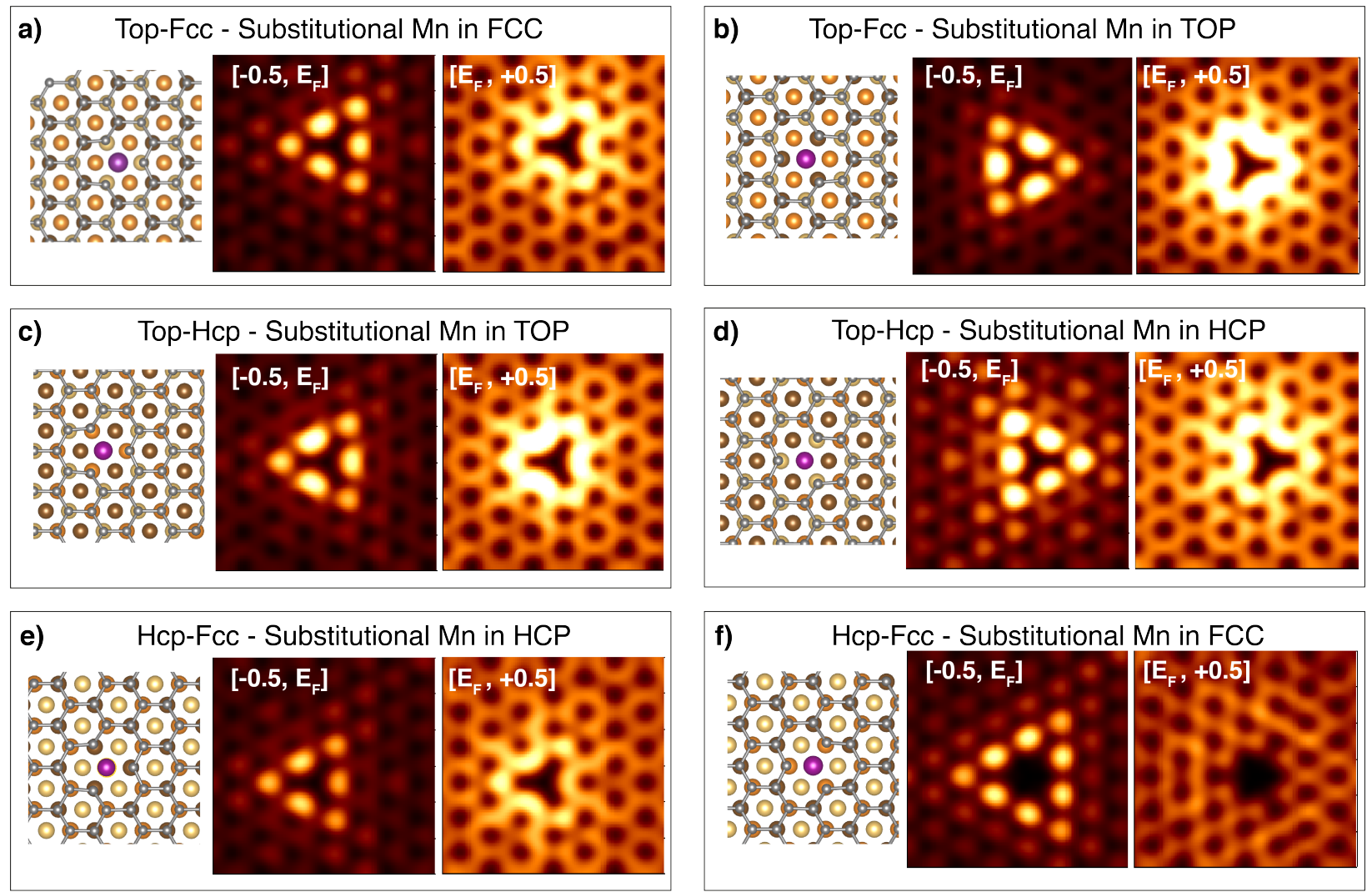

Figure S2: STM simulations for substitutional Mn in the three high-symmetry stacking configurations (TOP-FCC, TOP-HCP and HCP-FCC) and, for each stacking configuration, in each of the two possible graphene sublattices. All the simulations are performed with the same tip-surface distance $(3 \AA)$, with the same tip size $(1 \AA)$, and are plotted in the same contrast scale. The integration energy ranges are indicated $(0.5 \mathrm{eV}$ below and 0.5 above the Fermi level $\left.E_{F}\right)$. 
Table S1: Estimation of the substitutional Mn concentration.

\begin{tabular}{cccc}
\hline Micrograph & Area $\left(\mathrm{nm}^{2}\right)$ & \# of defects & Concentration \\
\hline 1 & 400 & 1 & $0.0071 \%$ \\
2 & 100 & 1 & $0.0284 \%$ \\
3 & 400 & 5 & $0.0355 \%$ \\
4 & 100 & 1 & $0.0284 \%$ \\
5 & 100 & 2 & $0.0568 \%$ \\
6 & 400 & 3 & $0.0213 \%$ \\
7 & 100 & 2 & $0.0568 \%$ \\
8 & 100 & 2 & $0.0568 \%$ \\
9 & 100 & 4 & $0.1136 \%$ \\
10 & 100 & 2 & $0.0568 \%$ \\
11 & 400 & 8 & $0.0568 \%$ \\
12 & 400 & 6 & $0.0426 \%$ \\
13 & 400 & 7 & $0.0497 \%$ \\
14 & 400 & 4 & $0.0284 \%$ \\
15 & 400 & 8 & $0.0568 \%$ \\
16 & 100 & 3 & $0.0852 \%$ \\
17 & 400 & 5 & $0.0355 \%$ \\
18 & 400 & 8 & $0.0568 \%$ \\
19 & 400 & 8 & $0.0568 \%$ \\
\hline
\end{tabular}

based on comparison with the DFT simulated STM topographies. The outwards configuration of substitutional Mn was rarely observed (STM micrograph in Figure S3(a), DFT simulation in Figure S1(c)). Moreover, four different intercalated forms could be discerned. Two single Mn intercalated configurations, in the HCP position (STM in Figure S3(b), DFT in Figure S1(d)) and in the FCC position (STM in Figure S3(c), DFT in Figure S1(e)) were observed, as well as a possible intercalated Mn cluster (Figure S3(d)). Our data further indicates that these three forms of intercalated Mn aggregate into intercalated Mn islands (Figure S3(e)) upon annealing at $\geq 600{ }^{\circ} \mathrm{C}$.

\section{Carbon single vacancy}

Although rare, objects resembling the DFT simulation for the carbon single vacancy (Figure S1(b)) appear in some micrographs (Figure S4), after annealing at $700{ }^{\circ} \mathrm{C}$, suggesting that a negligible concentration of vacancies may be formed upon high temperature annealing 

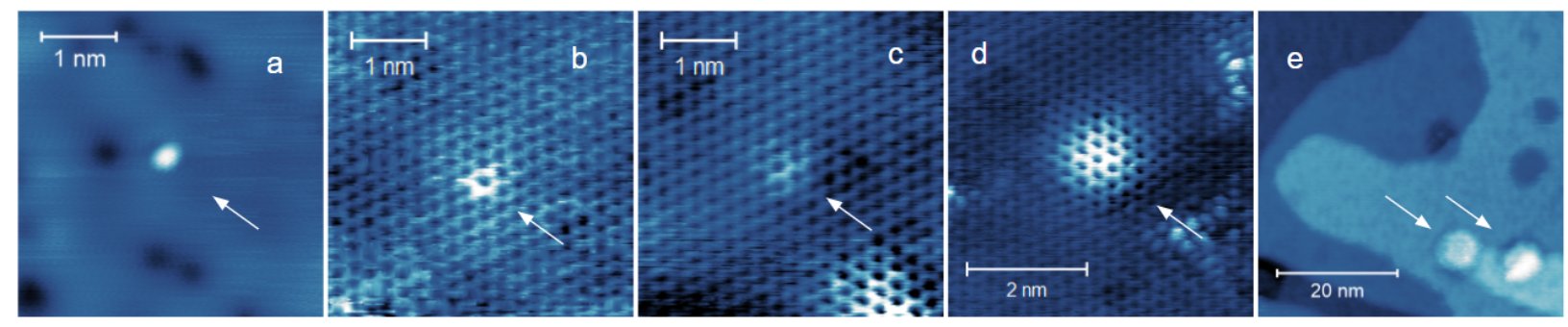

Figure S3: STM micrographs showing objects which can be attributed to other forms of $\mathrm{Mn}$ incorporation in graphene $/ \mathrm{Cu}(111)$, i.e. other than substitutional in the inwards configuration: (a) substitutional $\mathrm{Mn}$ in the outwards configuration $\left(V_{\text {sample }}=200 \mathrm{mV}, I_{\text {tun }}=\right.$ $900 \mathrm{pA})$; (b) single intercalated $\mathrm{Mn}$ in HCP position $\left(V_{\text {sample }}=-400 \mathrm{mV}, I_{\text {tun. }}=100 \mathrm{pA}\right)$; (c) single intercalated $\mathrm{Mn}$ in the FCC position $\left(V_{\text {sample }}=-400 \mathrm{mV}, I_{\text {tun. }}=100 \mathrm{pA}\right)$; (d) intercalated Mn cluster $\left(V_{\text {sample }}=-400 \mathrm{mV}, I_{\text {tun. }}=100 \mathrm{pA}\right)$; (e) intercalated $(\mathrm{Mn}$ or $\mathrm{Mn} / \mathrm{Cu}$ alloy) islands $\left(V_{\text {sample }}=-200 \mathrm{mV}, I_{\text {tun. }}=80 \mathrm{pA}\right)$. Micrographs (a) and (e) were measured after annealing at $600{ }^{\circ} \mathrm{C}$. Micrographs (b-d) were measured in the as-implanted state.

in UHV.

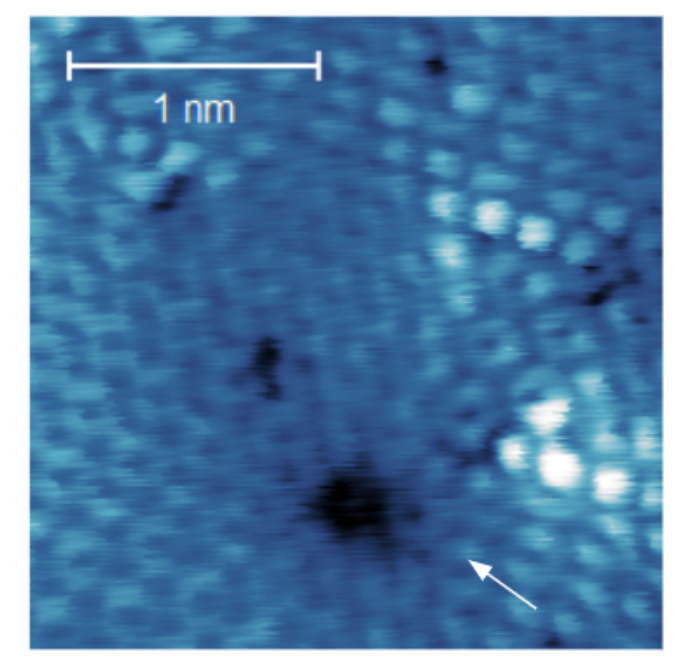

Figure S4: STM micrograph showing a possible carbon single vacancy, measured after $700{ }^{\circ} \mathrm{C}$ annealing $\left(V_{\text {sample }}=100 \mathrm{mV}, I_{\text {tun. }}=1.5 \mathrm{nA}\right)$

\section{Quantification of surface Mn, C and O}

To evaluate the effect of thermal annealing on the amount of Mn and contaminants present at the surface, XPS quantification analysis was performed on three core-levels: Mn 2 $\mathrm{p}_{3 / 2}, \mathrm{C}$ 1s, O 1s (spectra presented in Figure 1(c) and Figure S5). In order to estimate the relative 
surface concentrations, the following three parameters are typically considered: backgroundsubtracted peak area $(A)$, photoionization cross-section $(\sigma)$ (Table S2), and photoelectron escape depth. Given the similar photoelectron kinetic energies $\left(E_{k}\right)$ under our measurement conditions, we assumed the escape depth to be the same for the photoelectrons from the core-levels considered here; the error induced by this approximation is further decreased by the fact that all species are distributed within a very narrow depth range. As reference, we took the $\mathrm{C}$ signal in the pristine graphene sample, after annealing $700{ }^{\circ} \mathrm{C}$, as one monolayer equivalent (MLE) of carbon in graphene. All other concentrations were taken as relative to this $\mathrm{C}$ monolayer. For example, the surface concentration of $\mathrm{Mn}\left(c_{M n}\right)$, with respect to one C MLE, was estimated as:

$$
c_{M n}=\frac{A_{M n} / \sigma_{M n}}{A_{C, 700} / \sigma_{C, 700}}
$$

Table S3 compiles the concentration values obtained for Mn, C and O, for different annealing temperatures. We avoided measurements before thermal annealing, i.e. as-mounted in the UHV chamber after exposure to ambient conditions, since in that state the surface is inherently highly contaminated. Such adsorbed contaminants generally hinder the quantification of elements which are present underneath. Moreover, irradiation with high-flux X-rays may irreversibly modify the surface when such high concentrations of contaminants are present. The most striking feature is the decrease in Mn concentration upon $700{ }^{\circ} \mathrm{C}$, as discussed in the main text (Figure 1(c)). Moreover, the significant decrease in surface $\mathrm{O}$ in the pristine sample, from $425{ }^{\circ} \mathrm{C}$ to $700{ }^{\circ} \mathrm{C}$, confirms that surface contamination is significantly decreased. In the Mn implanted sample, the surface O content remains around $20 \%$, even after annealing at $700{ }^{\circ} \mathrm{C}$, indicating that surface oxidation may be enhanced by the Mn implantation and subsequent exposure to ambient, before the characterization steps. Most importantly, the C signal of the Mn-implanted sample (c), in particular after annealing at $700{ }^{\circ} \mathrm{C}$, preserves the signature of pristine graphene (a), consistent with the low level of disorder observed with Raman spectroscopy and STM. In short, and as also discussed in the main text, the (partial) removal of non-substitutional Mn components, combined with 
a cleaner graphene surface and low disorder, make the $700{ }^{\circ} \mathrm{C}$ annealed state particularly suitable for a more detailed study of Mn substitutionally doped graphene, which constitutes the focus of this article.

Table S2: Photoionization cross-sections.

\begin{tabular}{ccc}
\hline $\mathrm{C} 1 \mathrm{~s}$ & O $1 \mathrm{~s}$ & Mn $2 p_{3 / 2}$ \\
\hline $0.4247(h \nu=400 \mathrm{eV})$ & $0.3383(h \nu=650 \mathrm{eV})$ & $0.6746(h \nu=780 \mathrm{eV})$ \\
\hline
\end{tabular}

Table S3: Temperature dependence of $\mathrm{O}$ and $\mathrm{C}$ concentration on pristine graphene/ $\mathrm{Cu}(111)$.

\begin{tabular}{ccc}
\hline Element & $425^{\circ} \mathrm{C}$ annealed & $700^{\circ} \mathrm{C}$ annealed \\
\hline $\mathrm{O}(\% \mathrm{MLE})$ & 51.9 & 9.4 \\
$\mathrm{C}(\% \mathrm{MLE})$ & 77.6 & 100 \\
\hline
\end{tabular}

Table S4: Temperature dependence of $\mathrm{Mn}, \mathrm{O}$ and $\mathrm{C}$ concentration on $\mathrm{Mn}$ implanted graphene/Cu(111).

\begin{tabular}{cccc}
\hline Element & $200^{\circ} \mathrm{C}$ annealed & $425^{\circ} \mathrm{C}$ annealed & $700^{\circ} \mathrm{C}$ annealed \\
\hline Mn (\%MLE) & 2.3 & 2.2 & 1 \\
O (\%MLE) & 25.4 & 22.4 & 22.4 \\
$\mathrm{C}(\%$ MLE) & 81.5 & 86 & 84.6 \\
\hline
\end{tabular}

\section{Graphene domains}

The LEED image (Figure S6(a)) acquired at $52 \mathrm{eV}$ shows the hexagonal principle diffraction spots of $\mathrm{Cu}(111)$ substrate (innermost spot from the set of three spots, see inset) surrounded (on both sides) by the diffraction spots of graphene (outermost spots), forming slightly larger hexagons owing to a smaller real space lattice parameter of graphene $\left(a_{\mathrm{Gr}}=2.46\right.$ $\AA)$ as compared to the surface lattice parameter of $\mathrm{Cu}(111)\left(a_{\mathrm{Cu}}=2.56 \AA\right)$. By applying trigonometric functions to the reciprocal lattice parameters (indicated on the image), we can identify that the graphene lattice is not perfectly aligned with that of $\mathrm{Cu}(111)$. In particular, we observe the presence of two rotated sets of graphene domains (rotated by ca. $5.62 \pm 0.12^{\circ}$ with respect to each other), giving rise to two spots, i.e. rotated by $2.81 \pm 0.06^{\circ}$ with respect to $\mathrm{Cu}$ in opposite directions. The presence of these two sets of domains is also observed in the 

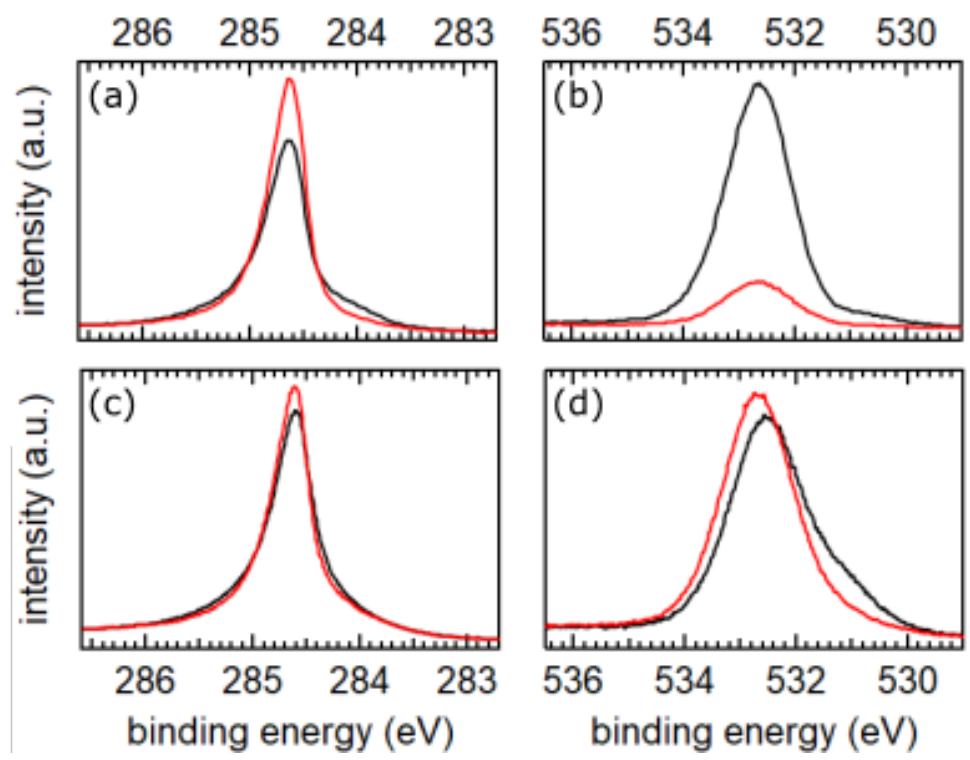

Figure S5: XPS measurements of $\mathrm{C}$ 1s $(\mathrm{a}, \mathrm{c})$ and $\mathrm{O}$ 1s $(\mathrm{b}, \mathrm{d})$ core-levels on pristine $(\mathrm{a}, \mathrm{b})$ and Mn-implanted (c, d) graphene/Cu(111), measured in the as-implanted state (black) and after annealing at $700{ }^{\circ} \mathrm{C}$ (red).

ARPES Fermi surface map (Figure S6(b)), consisting of a symmetric azimuthal scan of the first Brillouin zone near the $\mathrm{K}$ point, at a constant binding energy of $0.349 \mathrm{eV}$, near the Dirac point of pristine graphene sample. Two preferential domains can be identified, positioned at $\phi=164.11 \pm 0.05^{\circ}$ and $171.26 \pm 0.05^{\circ}$, respectively. The misalignment of $7.15 \pm 0.10^{\circ}$ between the two sets of domains, with the underlying $\mathrm{Cu}(111)$ in between $\left(3.57 \pm 0.05^{\circ}\right)$, is consistent with previously reported values (e.g. $3.4^{\circ}$, in).

\section{Identification of high-symmetry stacking regions}

As mentioned in the main text, DFT calculations yield Cu-graphene distances of $2.60 \AA$ for TOP-FCC, $2.70 \AA$ for TOP-HCP, and $2.87 \AA$ for HCP-FCC. We illustrate in Figure S7 the identification of the stacking regions within the Moiré superstructure, by extracting height profiles from the STM micrograph. On both profiles, height differences are observed which can be attributed to the three different stacking regions by comparison to the trend obtained in the DFT simulated heights, i.e. lower to higher from TOP-FCC, to TOP-HCP, to HCP-FCC. 

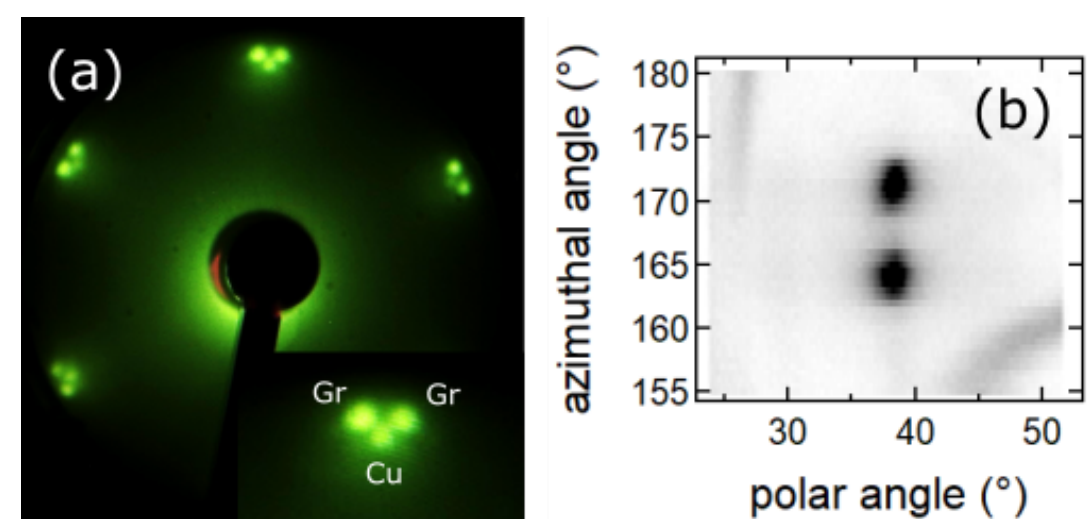

Figure S6: (a) LEED pattern of pristine graphene/Cu(111), observed at an electron beam energy of $52 \mathrm{eV}$. Inset: enlarged LEED spots to illustrate the two graphene spots and the sandwiched copper spot. (b) ARPES Fermi surface map of pristine graphene/Cu(111) near the $K$ point, obtained with $h \nu=34 \mathrm{eV}$, at a constant binding energy of $0.33 \mathrm{eV}$ (i.e. Dirac point).
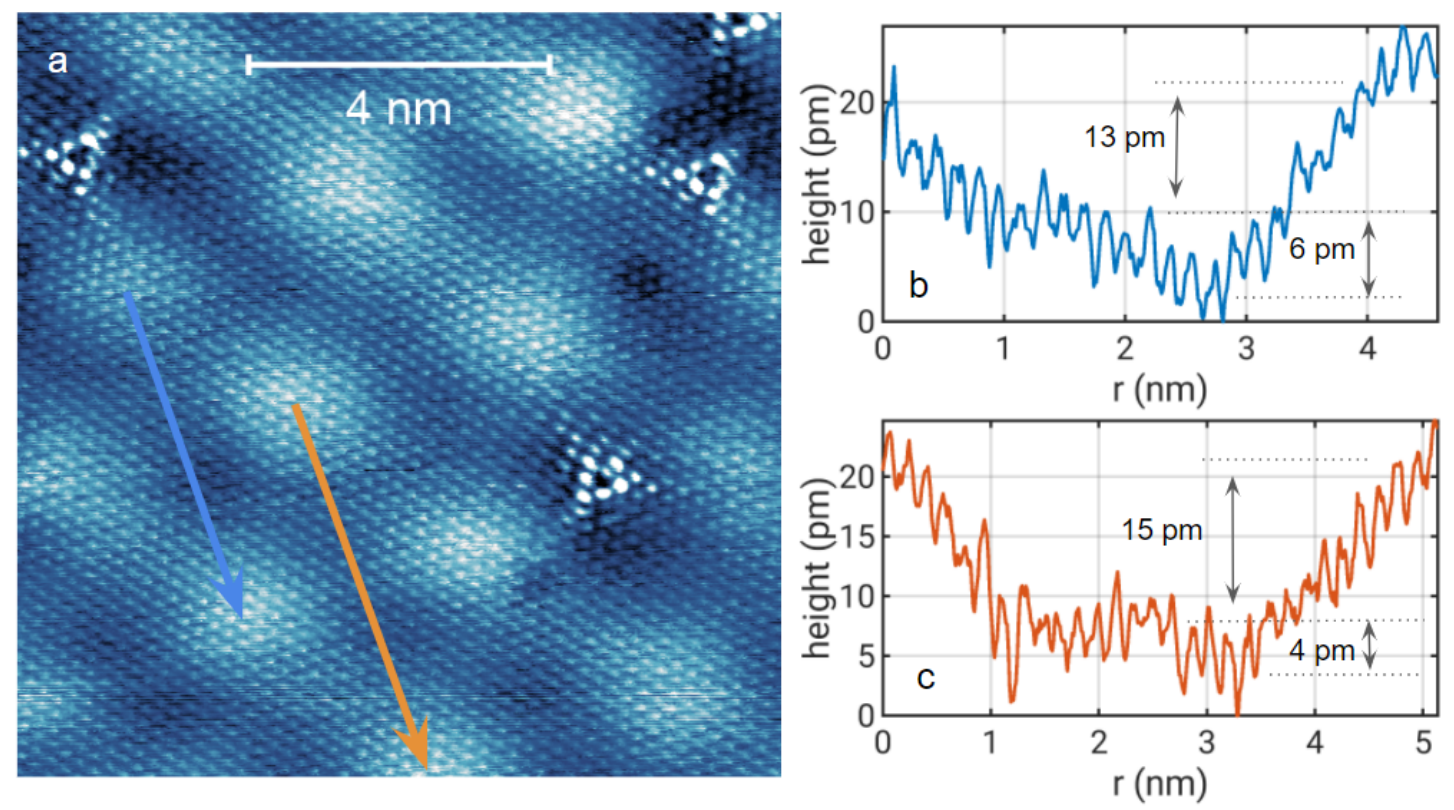

Figure S7: (a) STM micrograph showing the Moiré superstructure with two arrows displaying extracted profiles $\left(V_{\text {sample }}=100 \mathrm{mV}, I_{\text {tun }}=100 \mathrm{pA}\right.$ ), (b) extracted profile from HCP-FCC region to HCP-FCC region and (c) second extracted profile for statistics. 


\section{ARPES analysis: energy and momentum distribution curves}

The ARPES maps (p-polarization) were analyzed by extracting energy distribution curves (EDC) at constant $k_{\|}$, with a step of $0.01 \AA^{-1}$ (Figure $\left.4(\mathrm{e}, \mathrm{j})\right)$ and momentum distribution curves (MDC) at constant binding energy, with a step of $0.04 \mathrm{eV}$ (Figure S8). The extracted line profiles were fitted by single Lorentzian functions in order to determine their peak positions. The data points corresponding to those peaks in both the EDC and MDC line-profiles were then fitted with linear functions to find the two intercepts (valence and conduction bands) with the $K$ point (wavenumber $=1.7 \AA^{-1}$ ). The obtained gap value is defined as the difference between the two intercepts, resulting from the non-colinear valence and conduction bands. For both MDC and EDC analysis, we obtained the position of Dirac point (with respect to the Fermi level), defined as the midpoint in the gap. The values for the gap and Dirac point position quoted in the main text are based on the EDC analysis, as more commonly adopted. Here, in Table S5, we provide the results from the MDC analysis, for completeness. The Fermi velocity values quoted in the main text were obtained from the MDC analysis as:

$$
v_{F}=\frac{1}{\hbar} \frac{\partial E}{\partial k}
$$

where $\hbar=6.58 \times 10^{-16} \mathrm{eV} \cdot s$, and $\frac{\partial E}{\partial k}$ is taken as the slope of the conduction band, determined from the linear fit of the MDC local maxima near the Fermi level.

Table S5: MDC fitting. Estimated Dirac point position and gap opening.

\begin{tabular}{ccc}
\hline Sample & gap opening $(\mathrm{eV})$ & Dirac point position $(\mathrm{eV})$ \\
\hline pristine & $0.15 \pm 0.02$ & $0.36 \pm 0.01$ \\
Mn implanted & $0.09 \pm 0.02$ & $0.45 \pm 0.01$ \\
\hline
\end{tabular}



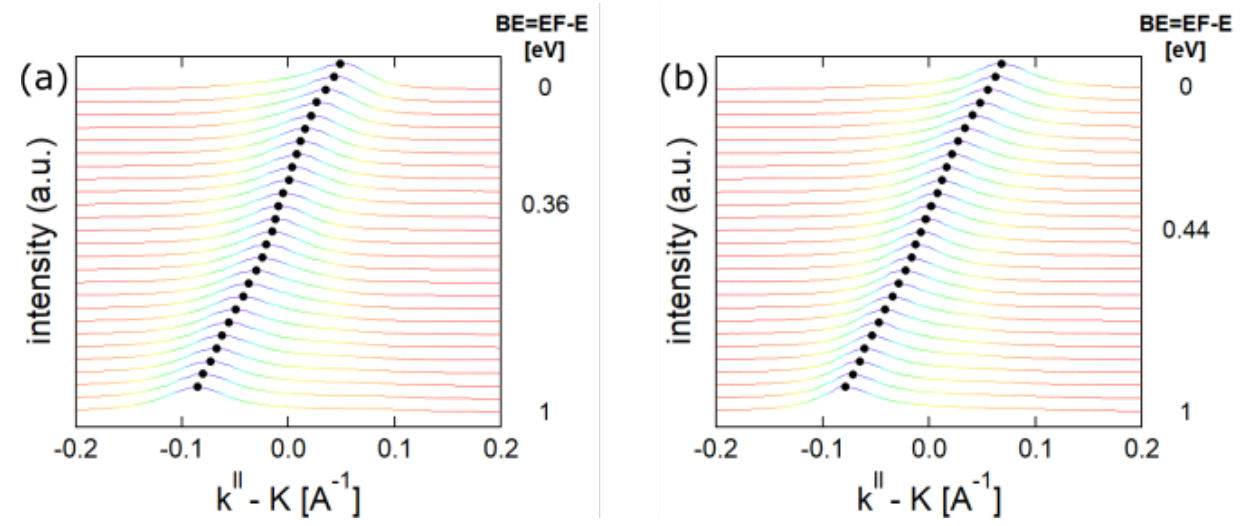

Figure S8: Momentum distribution curves (MDC) extracted from the p-polarization branch of the data in Figure 4(c, h), corresponding to pristine (a) and Mn-implanted (b) graphene $/ \mathrm{Cu}(111)$, with a binding energy $(\mathrm{BE})$ step of $0.04 \mathrm{eV}$. The dots mark the local maxima.

\section{Additional results from the DFT calculations}

In this section, we provide additional results concerning the DOS and band structure determined from the DFT calculations. We note that the structural model used for these calculations focuses on the local structural properties of the different stacking regions, aiming in particular at the identification of the defects observed with STM. In particular, the long range Moiré pattern observed for graphene $/ \mathrm{Cu}(111)$ is not accounted for in the calculations, since it would require much more computation-intensive calculations, with no additional insight of relevance for the present work.

Figure S9 shows the spin-resolved band structure of graphene $/ \mathrm{Cu}(111)$ with intercalated Mn (same configuration as in Figure S1(d)). The band structure shows a downshift of the Dirac cone with respect to the pristine graphene/ $\mathrm{Cu}(111)$ case due to charge transfer. The Dirac cone in pristine graphene $/ \mathrm{Cu}$ is found at $-0.62 \mathrm{eV}$ from the Fermi level (b) while in $\mathrm{Mn}$ intercalated graphene/ $\mathrm{Cu}$ it is at -0.86 and -0.64 for spin up and spin down components, respectively (c, d). As mentioned in the main text, it is plausible that the additional $n$-doping effect observed in the implanted sample (compared to pristine) is due to a non-vanishing concentration of such intercalated Mn atoms.

Figure S10 shows the spin-resolved band structure of graphene/Cu(111) with substitu- 
a)

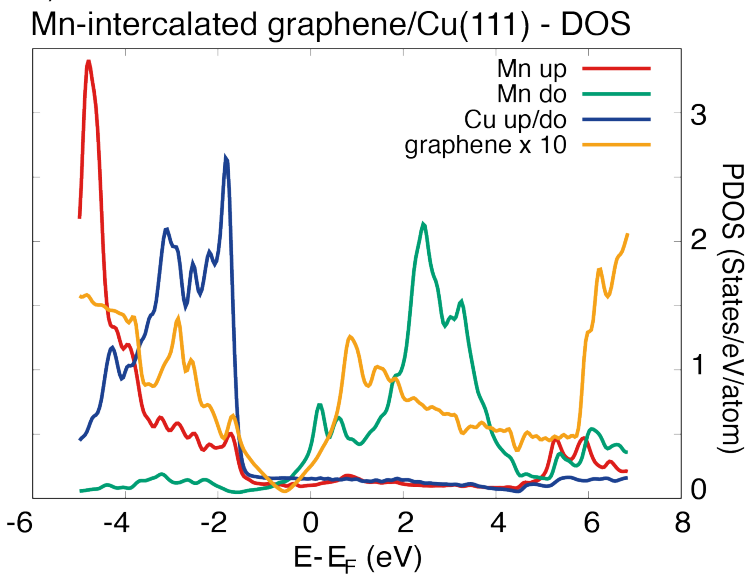

b)

Clean graphene/Cu(111) - minority spin

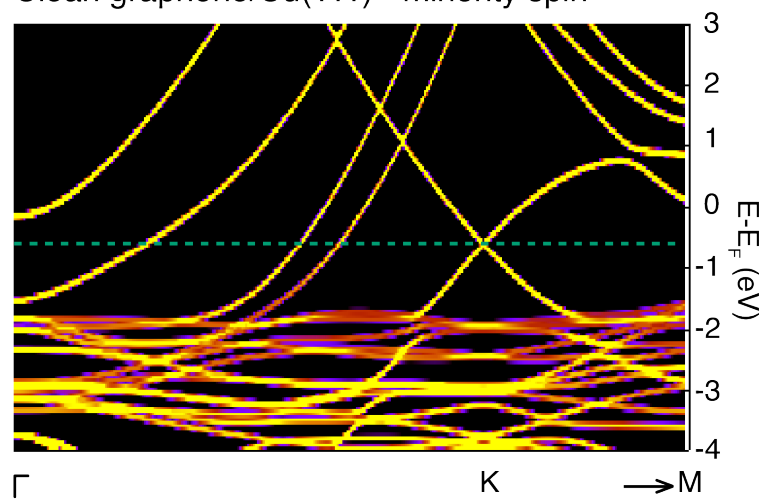

c)

Mn-intercalated graphene/Cu(111) - majority spin

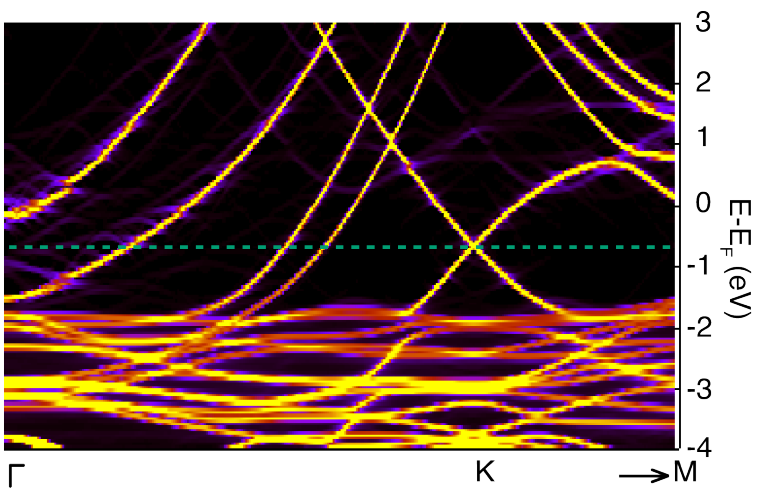

d)

Mn-intercalated graphene/Cu(111) - minority spin

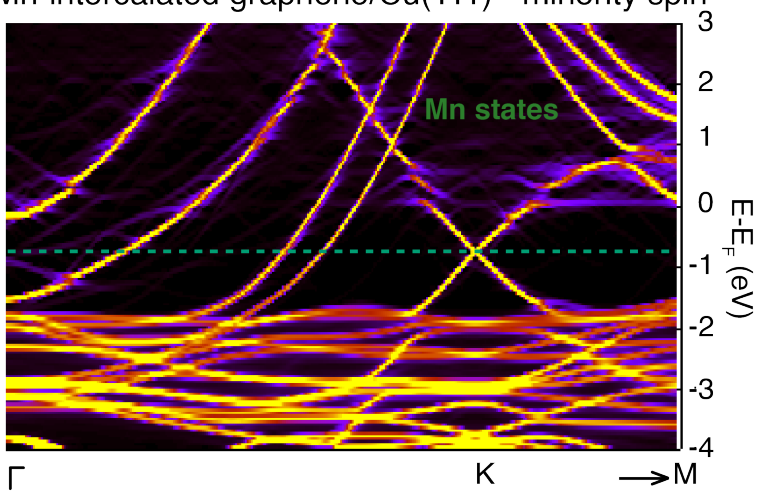

Figure S9: Spin-resolved projected density of states (a) and band structure of graphene/ $\mathrm{Cu}(111)$ with intercalated $\mathrm{Mn}$ atoms (c, d), compared to the band structure of pristine graphene $/ \mathrm{Cu}(111)(\mathrm{b})$. The PDOS are normalized to the number of $\mathrm{C}, \mathrm{Mn}, \mathrm{Cu}$ atoms. The $\mathrm{Mn} / \mathrm{C}$ ratio is $1 / 98$ and the $\mathrm{Mn} / \mathrm{Cu}$ ratio $1 / 295$. Green dashed lines in the band structures mark the position of the Dirac point. The band structure has been unfolded onto high symmetry paths of the $1 \times 1$ Brillouin zone. The position of Mn states, as deduced by the PDOS, is marked. 
a)

Mn substitutional graphene/Cu(111) DOS

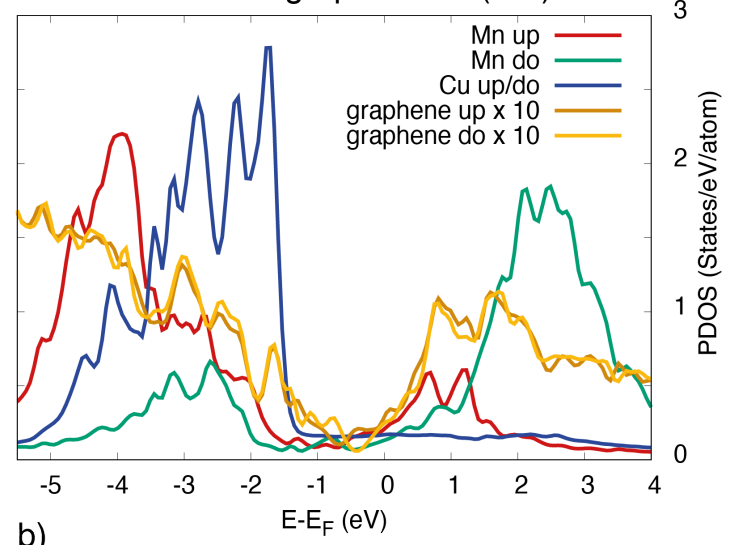

b)

Clean graphene/Cu (111) minority spin

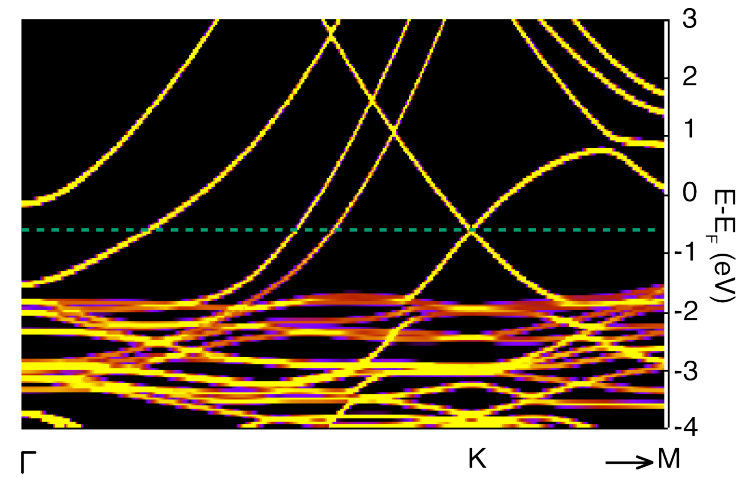

c) Mn substitutional graphene/Cu (111) majority spin

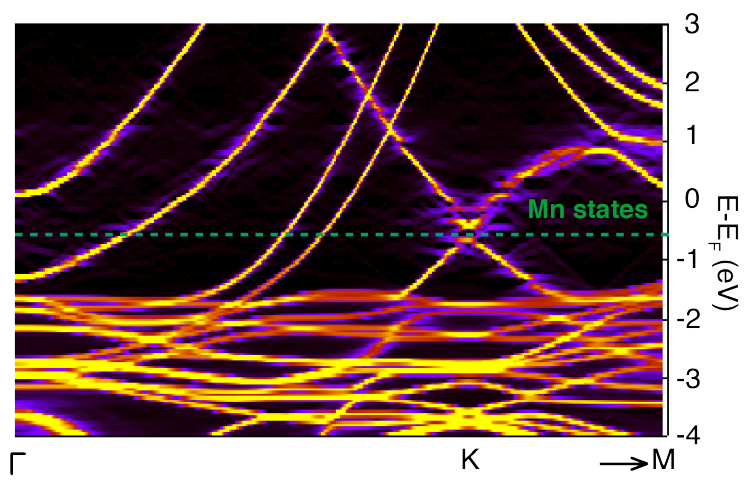

d)

Mn substitutional graphene/Cu (111) minority spin

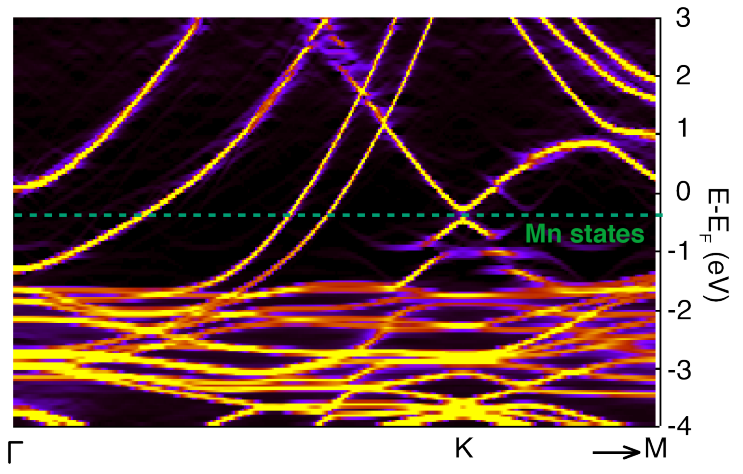

Figure S10: Spin-resolved projected density of states (a) and band structure of graphene $/ \mathrm{Cu}(111)$ with substitutional $\mathrm{Mn}$ atoms (c, d), compared to the band structure of pristine graphene $/ \mathrm{Cu}(111)$ (b). Green dashed lines in the band structures mark the position of the Dirac point. The $\mathrm{Mn} / \mathrm{C}$ ratio is $1 / 97$ and the $\mathrm{Mn} / \mathrm{Cu}$ ratio $1 / 295$. The band structure has been unfolded onto high symmetry paths of the $1 \times 1$ Brillouin zone. The position of Mn states, as deduced by the PDOS, is marked. 
tional Mn (inwards configuration). The Dirac cone energy in the substitutional case (-0.39 $\mathrm{eV}$ and $-0.54 \mathrm{eV}$ from the Fermi level for spin up and spin down components, respectively) is slightly higher than the pristine case $(-0.62 \mathrm{eV})$. This result further confirms that the additional $n$-doping observed experimentally is not due to substitutional Mn. A small gap at the Dirac cone is observed which is related to the carbon vacancy. The midgap state characteristic of the single $\mathrm{C}$ vacancy in graphene disappears upon incorporation of a $\mathrm{Mn}$ atom at the vacancy site.

In both cases, intercalated and substitutional, Mn states are superimposed to the graphene bands; the interaction between graphene and Mn states originates discontinuities in the Dirac cone (Figure S9 and S10). In particular, in the substitutional case (Figure S10), these are clearly visible just below the Fermi level, i.e. the region probed by ARPES. However, they are not observed in our experimental ARPES data, most likely due to the fact that the DFT calculations imply a periodic Mn arrangement, while the actual distribution in the sample is random. 


\section{Graphical TOC Entry}

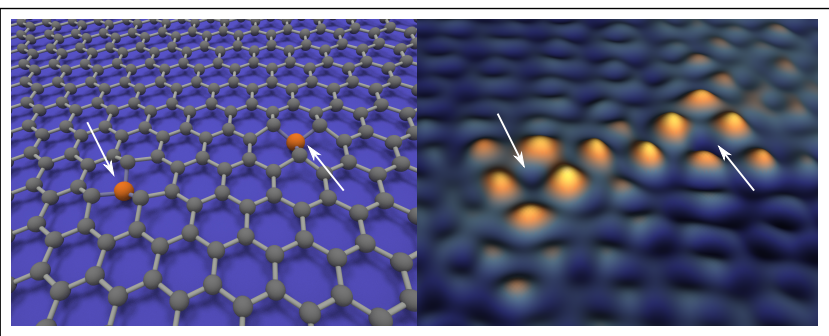

substitutional $\mathrm{Mn}$ atoms in opposite graphene sublattices 